\title{
Use and effects of implementation strategies for practice guidelines in nursing: a systematic review
}

Christine E. Cassidy ${ }^{1 *}$ (D), Margaret B. Harrison², Christina Godfrey², Vera Nincic ${ }^{3}$, Paul A. Khan ${ }^{3}$, Patricia Oakley ${ }^{4}$, Amanda Ross-White ${ }^{5}$, Hilary Grantmyre ${ }^{1}$ and lan D. Graham ${ }^{6}$

\begin{abstract}
Background: Practice guidelines can reduce variations in nursing practice and improve patient care. However, implementation of guidelines is complex and inconsistent in practice. It is unclear which strategies are effective at implementing guidelines in nursing. This review aimed to describe the use and effects of implementation strategies to facilitate the uptake of guidelines focused on nursing care.
\end{abstract}

Methods: We conducted a systematic review of five electronic databases in addition to the Cochrane Effective Practice and Organization of Care (EPOC) Group specialized registry. Studies were included if implementation of a practice guideline in nursing and process or outcome of care provided by nurses were reported. Two reviewers independently screened studies, assessed study quality, extracted data, and coded data using the EPOC taxonomy of implementation strategies. For those strategies not included in the EPOC taxonomy, we inductively categorized these strategies and generated additional categories. We conducted a narrative synthesis to analyze results.

Results: The search identified 46 papers reporting on 41 studies. Thirty-six studies used a combination of educational materials and educational meetings. Review findings show that multicomponent implementation strategies that include educational meetings, in combination with other educational strategies, report positive effects on professional practice outcomes, professional knowledge outcomes, patient health status outcomes, and resource use/ expenditures. Twenty-three of the 41 studies employed implementation strategies not listed within the EPOC taxonomy, including adaptation of practice guidelines to local context $(n=9)$, external facilitation $(n=14)$, and changes to organizational policy $(n=3)$. These implementation strategies also corresponded with positive trends in patient, provider, and health system outcomes.

Conclusions: Nursing guideline implementation may benefit from using the identified implementation strategies described in this review, including participatory approaches such as facilitation, adaptation of guidelines, and organizational policy changes. Further research is needed to understand how different implementation strategy components work in a nursing context and to what effect. As the field is still emerging, future reviews should also explore guideline implementation strategies in nursing in quasi or non-experimental research designs and qualitative research studies.

Keywords: Clinical practice guidelines, Nursing, Implementation, Implementation strategies, Systematic review

*Correspondence: ccassidy@dal.ca

${ }^{1}$ School of Nursing, Dalhousie University, 5860 University Ave., Halifax, NS B3H 4R2, Canada

Full list of author information is available at the end of the article permits use, sharing, adaptation, distribution and reproduction in any medium or format, as long as you give appropriate credit to the original author(s) and the source, provide a link to the Creative Commons licence, and indicate if changes were made. The images or other third party material in this article are included in the article's Creative Commons licence, unless indicated otherwise in a credit line to the material. If material is not included in the article's Creative Commons licence and your intended use is not permitted by statutory regulation or exceeds the permitted use, you will need to obtain permission directly from the copyright holder. To view a copy of this licence, visit http://creativecommons.org/licenses/by/4.0/. The Creative Commons Public Domain Dedication waiver (http://creativeco mmons.org/publicdomain/zero/1.0/) applies to the data made available in this article, unless otherwise stated in a credit line to the data. 


\section{Contributions to the literature}

- Findings from this review may inform nursing implementation researchers and practitioners in selecting strategies that facilitate the uptake of practice guidelines in nursing.

- This review identified additional implementation strategies similar to reviews in other health disciplines, including facilitation, guideline adaptation to the local context, changes to organizational policies, and use of a participatory approach.

- Future implementation science research in nursing, using qualitative, quantitative, and mixed methods research designs, is needed to help move the field beyond educational strategies and understand what works, for whom, and in what context.

\section{Background}

Implementing evidence into health care practice is essential for improving outcomes for patients, providers, and the health care system $[1,2]$. However, recent research estimates that on average, only $60 \%$ of care is consistent with evidence or consensus-based guidelines, $30 \%$ of care is either ineffective or low value, and $10 \%$ of care is harmful [3].

Nurses are the largest group of practitioners in health care systems, and thus have considerable potential to translate evidence into practice and influence patient and health system outcomes. A significant amount of international research, theory/framework design, policy, and education has been developed to advance the application of evidence-based nursing practice [4]. Notably, practice guidelines have emerged as a key tool for translating evidence into practice [5].

Practice guidelines are developed from systematic reviews of current evidence and offer graded recommendations that reflect best practice [5]. Guidelines have shown to be an effective strategy for improving health outcomes and processes of care in medicine [2, 6]. Despite these benefits, implementation of guidelines is both complex and varied [7-9]. Development of practice guidelines does not necessarily guarantee health care provider adoption and adherence in practice. A scoping review of barriers to guideline implementation in health care settings identified barriers related to personal factors (i.e., knowledge and attitudes), guidelinerelated factors (i.e., evidence, complexity, accessibility, and applicability of the guidelines), and external-factors (i.e., organizational constraints, resources, social and clinical norms) [10]. Tailored implementation strategies are needed to address these barriers and support uptake of guidelines in practice to impact patient and health system outcomes [11].

There is a need to fully identify the range of implementation strategies as well as the most effective strategies to improve the utility of guidelines in nursing practice. Previous systematic reviews have evaluated the effectiveness of implementation strategies, primarily in the medical domain $[2,12]$ and among allied health professionals, including rehabilitation [13], physiotherapy [14], dentistry [15], and pharmacy [16]. However, as Thompson and colleagues [17] point out, the nature and social structure of nursing work differs greatly from medicine and allied health professions. Nurses typically work in teams and in settings with procedures and protocols thus, not necessarily making sole decisions about care. Often the focus of implementation in nursing needs to be with a group and/or organization in addition to the individual [18]. As such, drawing conclusions about the effectiveness of interventions aimed at physician/allied health practice to guide implementation in nursing practice may not be appropriate. The purpose of this systematic review is to describe the use and effects of implementation strategies to facilitate uptake of guidelines focused on nursing care.

A recent systematic review examined implementation strategies used to implement nursing guidelines in daily practice [19]. This review identified 54 articles that employed a variety of implementation strategies with a majority of studies (68\%) reporting a positive effect on patient-related nursing outcomes or guideline adherence [19]. Our systematic review builds on these findings by replicating a similar approach and further examining the effect of implementation strategies on nurses' knowledge and practice, patient health status outcomes, and resource use/expenditures. Replication of systematic reviews is often disregarded, done poorly, or done unnecessarily [20]. Lack of or poor replication can lead to development and implementation of policies, guidelines, or practices that are based on weak evidence. Karunananthan et al. [21] describes two types of systematic review replication, including (a) the repetition of the same population, intervention, comparison, or outcome (PICO) using the same or very similar methods to a previous review, or (b) broadening or narrowing the PICO of a previous review. Our systematic review employs the second type of replication whereby the PICO is overlapping with the Spoon et al. [19] review but with a broader outcome focus and narrower study design focus. Collectively, these reviews add to the knowledge base on effective guideline implementation strategies in nursing. 


\section{Methods}

This review protocol was originally registered with Cochrane Effective Practice and Organization of Care (EPOC). The search strategy and screening methods followed Cochrane systematic review and meta-analysis guidance (see Supplemental File 1 for our a priori EPOC review methods). This initial review generated a heterogeneous set of papers with a wide variety of implementation strategies (i.e., mode of delivery, dose, frequencies), and outcome measures. Although the data described specific strategies, it was not helpful for interpreting the overall effectiveness and utility of implementation strategies in nursing or the next steps for future research in nursing implementation. We were challenged to make sense of the data in a useful way for practice and to move the state of the science forward. Following this initial analysis, we identified several ad hoc discoveries as equally, if not more, important for nursing implementation science and future reviews. We therefore conducted a narrative review of the included papers with a different lens than originally intended to more fully describe the findings related to implementation strategies for nursing. The following methods and results reflect this narrative approach to the review. We completed the review in accordance with the Preferred Reporting for Systematic Reviews and Meta-Analyses (PRISMA) statement and checklist. See Supplemental File 1 for deviations from the initial protocol.

\section{Information sources and search strategy}

Five electronic databases (Medline, EMBASE, CINAHL, PsycINFO, AMED) and the Cochrane EPOC registry were systematically searched using a search strategy developed with nursing library scientists at Queen's University. The team used search terms and medical subject headings (MeSH) relevant to "clinical guidelines" AND "implementation" AND "nursing" AND "randomized controlled trial" (Supplemental File 2). The search was run up to September 30th, 2020 with no restrictions. We also used the search strategies developed by Grimshaw and colleagues [22] for their investigation of the effectiveness and efficiency of guideline dissemination and implementation strategies in the context of medicine. These search strategies were adjusted to focus on nursing and rerun against MEDLINE, CINAHL, and EMBASE. We also scanned reference lists of papers identified for inclusion for any additional references not captured.

Eligibility criteria.

Inclusion criteria for this review were that studies had to (a) be written in English and published in a peerreviewed journal, (b) use a randomized controlled trial (RCT) design, and (c) evaluate the implementation of a guideline targeted to nurses or a multidisciplinary team with a focus on nursing outcomes. Implementation strategies are defined as "methods or techniques used to enhance the adoption, implementation, and sustainability of a clinical program or practice" [23]. Guidelines are defined as "systematically developed statements to assist practitioner and patient decisions about appropriate health care for specific clinical circumstances" [17]. A more recent definition includes benefits and harms (but no longer includes the goal of assisting practitioner and patient decisions); "Clinical practice guidelines are statements that include recommendations intended to optimize patient care that are informed by a systematic review of evidence and an assessment of the benefits and harms of alternative care options" [5]. Substitute terms for practice guidelines included "protocol," "standard," "algorithm," and "clinical pathway." The primary comparator was "usual practice" or "usual care" which indicates no distinct implementation strategy was used to change nursing practice or a traditional approach to dissemination was used (e.g., guidelines were available for use in practice setting, nurses received a copy of the guidelines). Categories of nurses included advanced practice nurses $(\mathrm{APN})$; nurse practitioners (NPs); clinical educators (CE); clinical nurse specialists (CNS); registered nurses (RN); and licensed practical nurses (LPN)/registered practical nurses (RPN).

We excluded studies that were (a) not a RCT design; (b) abstracts, conference proceedings; (c) targeting implementation strategies to patients, administrators, and other health care providers (when outcomes could not be attributed to nurses); and (d) not focused on implementing practice guidelines.

\section{Outcomes}

Outcomes of interest were primary outcomes focused on process or outcome of care provided by nursing professionals. Outcomes were grouped into five categories (professional knowledge, professional practice, patient health status outcomes, resource use, and expenditures). Professional knowledge outcomes related to level of nursing knowledge. Professional practice outcomes related to nursing process of care (i.e., adherence to practice guidelines). Patient health status outcomes included physical health and treatment outcomes (i.e., pain, quality of life, incontinence). Economic outcomes related to resource use and measured costs and cost savings (expenditures) associated with guideline implementation [24].

\section{Study selection}

Two reviewers independently screened the titles and abstracts for inclusion in Covidence [25]. Next, two reviewers independently screened the full-text articles against the inclusion criteria. When there was 
disagreement, a third and independent reviewer assessed the study. Where separate papers reported on different aspects of the same study (for example, one paper describing the effect of the intervention on professional practice and another, the effect on patient health status outcomes), we treated them as one study with two companion papers.

\section{Data abstraction}

Two reviewers independently abstracted data from included studies using a standardized form adapted from the EPOC data collection checklist. The following data were extracted from each eligible full-text study: (a) study design, (b) participants, (c) setting, (d) data collection methods, (e) practice guideline, (f) use of theory, (g) types of implementation strategies, (h) outcome measures, and (i) study results. Two reviewers independently piloted the data extraction form with two studies and revisions were made. Where there was disagreement in data abstraction, a third and independent reviewer assessed the study and resolved the conflict. For those studies where separate papers reported on different outcomes of the same study, only one data abstraction template was completed.

\section{Categorization of implementation strategies}

We initially used the Cochrane Effective Practice and Organization Care Review Group (EPOC) taxonomy to describe the implementation strategies included in this review. The EPOC taxonomy has been used in previous reviews of implementation strategies with a similar scope but with different practice settings $[16,26]$. To classify implementation strategies, we started by deductively categorizing strategies into the EPOC taxonomy's section on implementation strategies [27]. Next, for those strategies not included in the EPOC taxonomy, we used an inductive thematic analysis approach to group these strategies and generate additional implementation strategy categories [28].

\section{Study quality}

Two reviewers independently assessed the risk of bias using the EPOC Risk of Bias 2.0 checklist in Covidence [29]. For each of the included studies, risk of bias was assessed as a judgment of high, low, or unclear risk across nine domains. Discrepancies were resolved by consensus with a third reviewer when necessary.

\section{Data analysis}

We conducted a narrative synthesis after identifying methodological and clinical heterogeneity in the studies of this review; this indicated that meta-analysis was not appropriate. A narrative synthesis allows for a description of implementation strategies and their effects in achieving outcomes for guideline implementation in nursing [30]. The frequency of each EPOC taxonomy category, outcome measure category, and outcome effect is reported. For outcome effect, we describe whether the outcomes were reported as a statistically significant positive effect or had no effect. Separate comparisons were made for categories of implementation strategies and compared to grouped study results to determine whether they were related to positive and significant improvement in: professional knowledge outcomes, professional practice outcomes, patient health status outcomes, resource use outcomes, and/or expenditure outcomes.

\section{Results}

Study selection

All database searches and hand-searching of reference lists of included studies yielded a total of 38,172 citations. No studies were found using the Grimshaw et al. (22) search strategy. After removal of 4890 duplicates, 33,282 citations were screened, and 924 potential articles were identified. From this set, 878 articles did not meet our inclusion criteria and were excluded from analyses. The majority were excluded for not using an experimental study design (RCT), not implementing a guideline, and not including a nursing population. Fortyone studies (reported in 46 papers due to five companion reports) met final inclusion criteria (Fig. 1). All studies included were conducted between January 1996 and September 2020.

\section{Study characteristics}

Full details of study-level characteristics are described in Table 1. The final set included 41 randomized controlled trials (RCTs), of which 24 were reported to be cluster randomized controlled trials (cRCT). The number of intervention arms ranged from two to three (two arms $=36$ studies, three arms $=5$ studies). In total, the 41 studies included a minimum of 5429 nurses (2 studies did not specify the number of participating nurses). In addition, a minimum of 231,218 patients were implicated in 27 studies, with 14 studies not reporting on the number of participating patients.

Eighteen studies were conducted in North America (16 in the USA, 2 in Canada), 5 in the UK, 13 in continental Europe (4 in the Netherlands, 3 in Germany, 2 in Finland, 2 in Italy, 1 in Austria, 1 in Belgium), 2 in South Africa, and 3 in Asia (1 in Thailand, 1 in China, and 1 in South Korea) (Table 1).

Key guideline topics included respiratory (asthma, pneumonia) $(n=7)$, heart conditions $(n=5)$, pain $(n=3)$, cancer $(n=2)$, diabetes $(n=2)$, pressure ulcer prevention $(n=2)$, maternity care $(n=2)$, and urinary incontinence $(n=2)$ (Table 1). Various nursing practices and health 

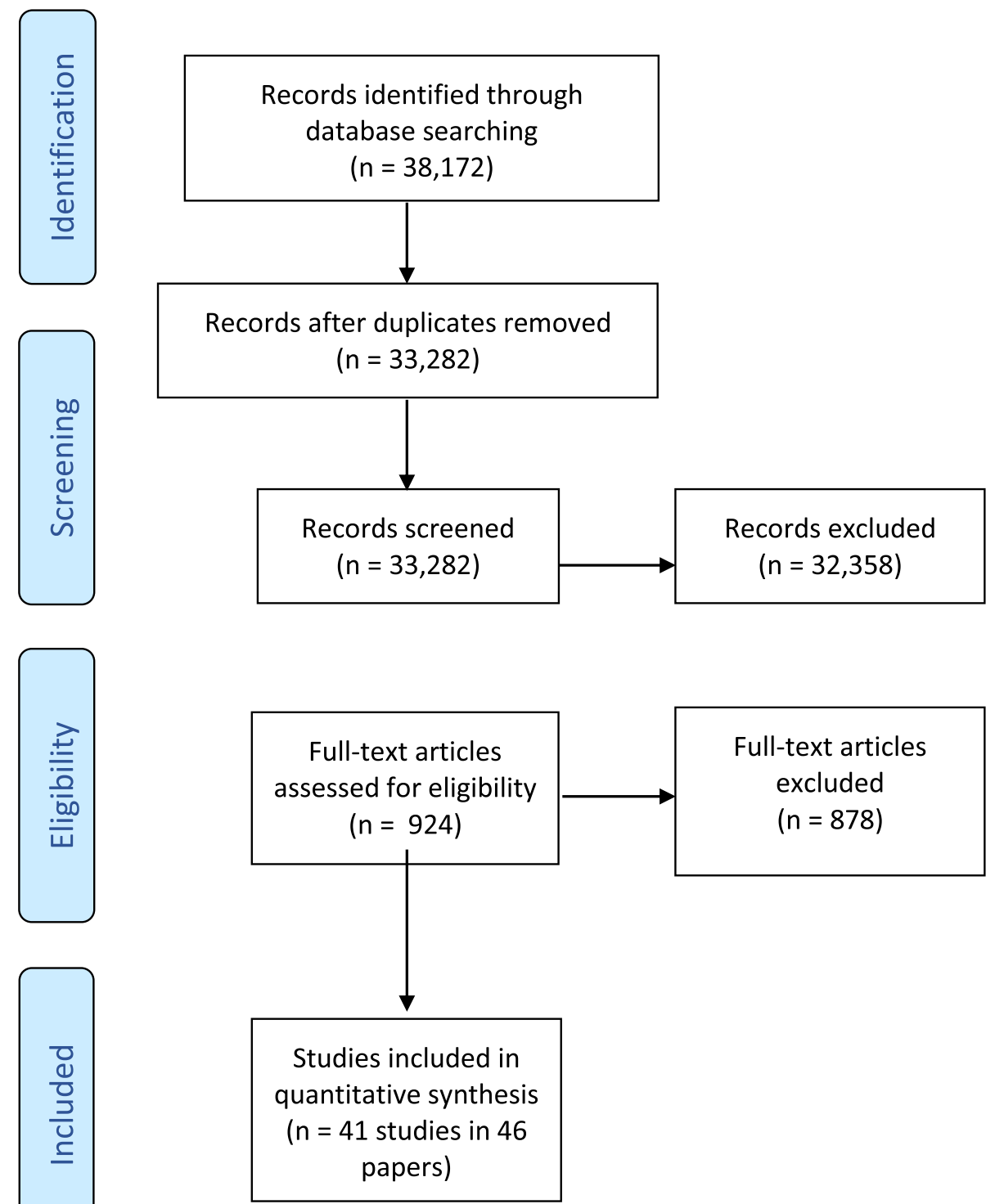

Fig. 1 PRISMA flow diagram

care settings were identified, including intensive care units, nursing homes, and in the community. The use of theory to inform the intervention and/or implementation strategy was used in 17 studies, including identifying behavioral and environmental determinants, identifying intervention targets, selecting implementation methods and delivery strategies, and informing measurement and evaluation.

\section{Risk of bias assessments}

Methodological quality of the studies varied (Table 2). Most of the studies had a low risk of bias in their allocation concealment $(n=33,80 \%)$, baseline outcome measurements $(n=36,88 \%)$ and baseline characteristic similar $(n=32,78 \%)$, as well as a low risk in other sources of bias $(n=37,90 \%)$. Two-thirds of the studies completed high-quality (low risk) random sequence generations $(n=27,66 \%)$, while a number of studies had an unclear risk of randomization techniques $(n=14,34 \%)$. Knowledge of the allocated interventions was adequately prevented in half the studies $(n=21,50 \%)$, while in the rest of the cases it was not clear $(n=19,46 \%)$, or not prevented $(n=2,5 \%)$. Compared to other categories, a higher level of risk was found for incomplete outcome 
Table 1 Characteristics of included studies

\begin{tabular}{|c|c|c|c|c|c|}
\hline Author & Study design & $\begin{array}{l}\text { Participants: } \\
\text { healthcare } \\
\text { providers }\end{array}$ & Participants: patients & Setting & Practice guidelines \\
\hline Ammerman, 2003 (USA) & $\mathrm{RCT}$ & Nurses & $\begin{array}{l}\text { Food for Heart program } \\
\text { patient }\end{array}$ & Hospital—Outpatient & $\begin{array}{l}\text { Dietary counseling for } \\
\text { hypercholesterolemia }\end{array}$ \\
\hline Charrier, 2008 (Italy) & $\mathrm{CRCT}$ & Nurses & Adult inpatient & Hospital_Inpatient & $\begin{array}{l}\text { Prevention of pressure } \\
\text { lesions and the manage- } \\
\text { ment of peripheral and } \\
\text { central venous catheters }\end{array}$ \\
\hline Cheater, 2006 (UK) & CRCT & Nurses & Adult outpatient & Hospital_Outpatient & $\begin{array}{l}\text { Management of urinary } \\
\text { incontinence }\end{array}$ \\
\hline Daniels, 2005 (USA) & $\mathrm{RCT}$ & Nurses, Physicians & Adult outpatient & Hospital—Outpatient & Asthma care management \\
\hline Day, 2001 (UK) & $\mathrm{RCT}$ & Nurses & $\begin{array}{l}\text { Adult intensive care } \\
\text { inpatient }\end{array}$ & Inpatient-Hospital & Endotracheal suctioning \\
\hline Donati, 2020 (Italy) & $\mathrm{CRCT}$ & Nurses & Medical-surgical & Inpatient_-Hospital & Standard precautions \\
\hline Elliott, 1997 (USA) & CRCT & Nurses & Oncology patient & $\begin{array}{l}\text { Community Primary Care } \\
\text { Clinic }\end{array}$ & Cancer pain management \\
\hline Evans, 1997(USA) & $\mathrm{CRCT}$ & Nurses, Physicians & Pediatric inpatient & Hospital—Outpatient & Asthma care management \\
\hline $\begin{array}{l}\text { Fairall, 2005, } 2010 \text { (South } \\
\text { Africa) }\end{array}$ & CRCT & NP & Adult outpatient & $\begin{array}{l}\text { Community Primary Care } \\
\text { Clinic }\end{array}$ & $\begin{array}{l}\text { Tuberculosis case detection } \\
\text { and respiratory care }\end{array}$ \\
\hline Feldman, 2004 (USA) & $\mathrm{RCT}$ & Nurses & $\begin{array}{l}\text { Chronic heart failure } \\
\text { patient }\end{array}$ & Hospital—Outpatient & Heart failure management \\
\hline Friese 2019 (USA) & CRCT & Nurses & Oncology patients & Hospital_Inpatient & Hazardous Drug Handling \\
\hline $\begin{array}{l}\text { Haegdorens, } 2018 \text { \& } 2019 \\
\text { (Belgium) }\end{array}$ & $\mathrm{CRCT}$ & Nurses & Medical-surgical & Hospital_-inpatient & Early warning score \\
\hline $\begin{array}{l}\text { Harrison, } 2000 \text { (South } \\
\text { Africa) }\end{array}$ & $\mathrm{RCT}$ & Nurses & Community clinic patient & Community & $\begin{array}{l}\text { Sexually transmitted infec- } \\
\text { tion management }\end{array}$ \\
\hline Hödl, 2019 (Austria) & CRCT & Nurses & Nursing home resident & Nursing Home & $\begin{array}{l}\text { Urinary incontinence man- } \\
\text { agement }\end{array}$ \\
\hline Hodnett, 1996 (Canada) & CRCT & Nurses & $\begin{array}{l}\text { Labor and delivery } \\
\text { patients }\end{array}$ & Hospital_Inpatient & $\begin{array}{l}\text { Intrapartum nursing } \\
\text { practice }\end{array}$ \\
\hline Jansson, 2014(Finland) & $\mathrm{RCT}$ & Nurses & $\begin{array}{l}\text { Adult intensive care } \\
\text { inpatient }\end{array}$ & Hospital_Inpatient & $\begin{array}{l}\text { Prevention of ventilator- } \\
\text { associated pneumonia }\end{array}$ \\
\hline $\begin{array}{l}\text { Jansson, 2016a, 2016b } \\
\text { (Finland) }\end{array}$ & $\mathrm{RCT}$ & Nurses & $\begin{array}{l}\text { Adult intensive care } \\
\text { inpatient }\end{array}$ & Hospital_Inpatient & $\begin{array}{l}\text { Prevention of ventilator- } \\
\text { associated pneumonia }\end{array}$ \\
\hline $\begin{array}{l}\text { Kalinowski, } 2015 \text { (Ger- } \\
\text { many) }\end{array}$ & $\mathrm{RCT}$ & Nurses & Nursing home resident & Nursing Home & $\begin{array}{l}\text { Nonpharmacological pain } \\
\text { management }\end{array}$ \\
\hline Kaner, 2003 (UK) & CRCT & Nurses & Adult outpatient & $\begin{array}{l}\text { Community Primary Care } \\
\text { Clinic }\end{array}$ & Brief alcohol intervention \\
\hline Köpke, 2012 (Germany) & CRCT & Nurses & Nursing home resident & Nursing Home & Use of physical restraint \\
\hline Lozano, 2004 (USA) & $\mathrm{RCT}$ & Nurses, Physicians & $\begin{array}{l}\text { Pediatric, asthmatic } \\
\text { patient }\end{array}$ & Hospital—Outpatient & $\begin{array}{l}\text { Pediatric chronic asthma } \\
\text { care }\end{array}$ \\
\hline Mayou, 2002 (UK) & $\mathrm{RCT}$ & Nurses & $\begin{array}{l}\text { Adult heart failure inpa- } \\
\text { tient }\end{array}$ & Hospital_Inpatient & $\begin{array}{l}\text { Early rehabilitation after } \\
\text { myocardial infarction }\end{array}$ \\
\hline McDonald, 2005 (USA) & $\mathrm{RCT}$ & Nurses & Adult outpatient & Hospital—Outpatient & Pain management \\
\hline Moon, 2015 (South Korea) & $\mathrm{RCT}$ & Nurses & $\begin{array}{l}\text { Adult intensive care } \\
\text { inpatient }\end{array}$ & Hospital_Inpatient & Delirium prevention \\
\hline Murtaugh, 2005 (USA) & $\mathrm{RCT}$ & Nurses & $\begin{array}{l}\text { Adult cardiology outpa- } \\
\text { tient }\end{array}$ & Hospital—Outpatient & $\begin{array}{l}\text { Heart failure disease man- } \\
\text { agement }\end{array}$ \\
\hline Naylor, 2004 (USA) & $\mathrm{RCT}$ & Nurses & Adult cardiology inpatient & Hospital_Inpatient & $\begin{array}{l}\text { Transitional care of older } \\
\text { adults hospitalized with } \\
\text { heart failure }\end{array}$ \\
\hline $\begin{array}{l}\text { Noome, } 2017 \text { (Nether- } \\
\text { lands) }\end{array}$ & $\mathrm{RCT}$ & Nurses & Adult inpatient & Hospital_Inpatient & Nursing end-of-life care \\
\hline
\end{tabular}


Table 1 (continued)

\begin{tabular}{|c|c|c|c|c|c|}
\hline Author & Study design & $\begin{array}{l}\text { Participants: } \\
\text { healthcare } \\
\text { providers }\end{array}$ & Participants: patients & Setting & Practice guidelines \\
\hline Pagaiya, 2005 (Thailand) & $\mathrm{RCT}$ & Nurses & $\begin{array}{l}\text { Adult and pediatric } \\
\text { outpatient }\end{array}$ & $\begin{array}{l}\text { Community Primary Care } \\
\text { Clinic }\end{array}$ & $\begin{array}{l}\text { Children: Acute respiratory } \\
\text { infection and diarrhea } \\
\text { Adults: Diazepam prescrib- } \\
\text { ing and standard manage- } \\
\text { ment of diabetes }\end{array}$ \\
\hline Parker, 1995(USA) & $\mathrm{RCT}$ & Nurses, NP & $\begin{array}{l}\text { Adult, long term care } \\
\text { patient }\end{array}$ & Long-term care facility & Diabetes management \\
\hline Premaratne, 1999 (UK) & $\mathrm{RCT}$ & Nurses & Community clinic patient & Health care clinic & Asthma management \\
\hline Rood, 2005 (Netherlands) & $\mathrm{RCT}$ & Nurses & Adult, inpatient & Hospital_Inpatient & Glucose regulation \\
\hline Ruijter, 2018 (Netherlands) & $\mathrm{RCT}$ & Nurses & Adult, outpatient & $\begin{array}{l}\text { Community Primary Care } \\
\text { Clinic }\end{array}$ & Smoking cessation \\
\hline $\begin{array}{l}\text { Snelgrove-Clarke, } 2015 \\
\text { (Canada) }\end{array}$ & $\mathrm{RCT}$ & Nurses & $\begin{array}{l}\text { Adult, low risk labor and } \\
\text { delivery patient }\end{array}$ & Hospital_Inpatient & Fetal health surveillance \\
\hline $\begin{array}{l}\text { Titler, 2009; Brooks, } 2008 \\
\text { (USA) }\end{array}$ & $\mathrm{RCT}$ & Nurses, Physicians & Older adults & Hospital_Inpatient & Acute pain management \\
\hline Tjia, 2015 (USA) & $\mathrm{CRCT}$ & Nurses & Nursing home residents & Long term care & Antipsychotic prescribing \\
\hline Vallerand, 2004 (USA) & $\mathrm{RCT}$ & Nurses & Adult outpatient & Hospital_Outpatient & Cancer pain management \\
\hline $\begin{array}{l}\text { Van Gaal, 2011a; } 2011 \mathrm{~b} \\
\text { (Netherlands) }\end{array}$ & $\mathrm{RCT}$ & Nurses & Older adults & $\begin{array}{l}\text { Long term care and Hospi- } \\
\text { tals -inpatient }\end{array}$ & $\begin{array}{l}\text { Patient care guidelines to } \\
\text { prevent adverse events } \\
\text { including: pressure ulcers, } \\
\text { urinary tract infections and } \\
\text { falls }\end{array}$ \\
\hline $\begin{array}{l}\text { VonLengerke, } 2017 \\
\text { (Germany) }\end{array}$ & $\mathrm{RCT}$ & Nurses, Physicians & $\begin{array}{l}\text { Adult intensive care } \\
\text { inpatient }\end{array}$ & Hospital_Inpatient & Hand hygiene \\
\hline Weiss, 2019 (USA) & $\mathrm{CRCT}$ & Nurses & Adults, medical surgical & Hospital_Inpatient & $\begin{array}{l}\text { Discharge Readiness Assess- } \\
\text { ment }\end{array}$ \\
\hline Wright, 1997 (USA) & $\mathrm{RCT}$ & Nurses & Adult inpatient & Hospital_Inpatient & $\begin{array}{l}\text { Universal precautions- } \\
\text { related behaviors }\end{array}$ \\
\hline Zhu, 2018 (China) & $\mathrm{RCT}$ & Nurses & Adult, outpatient & $\begin{array}{l}\text { Community Primary Care } \\
\text { Clinic }\end{array}$ & Hypertension management \\
\hline
\end{tabular}

data $(n=11,27 \%)$ and in protection against contamination $(n=7,17 \%)$; however, the majority were low risk of bias in these categories $(n=31,76 \%$ and $n=29,71 \%$, respectively). Lastly, the majority of studies had unclear $(n=18,44 \%)$ and low risk of bias $(n=24,59 \%)$ for selective outcome reporting.

\section{Implementation strategies used}

Table 3 reports on implementation strategies used across all studies. Of the 41 studies, a total of 152 strategies were used. Multi-component implementation strategies were most commonly used ( $n=36$ studies) with only five studies reporting single component strategies.

Of the three categories of EPOC implementation strategies, all studies reported interventions targeted at healthcare workers (Table 3 ). The most frequently used strategies to target nursing guideline implementation were educational strategies (i.e., educational meetings $(n=33)$, educational materials $(n=27)$, and educational outreach visits $(n=12))$. Audit and feedback strategies were used in 11 studies.
A wide range of educational strategies were used which highlights the challenge of classifying these interventions under the same heading (Table 4). Many studies used training sessions that included lectures, discussions, and video presentations to provide guideline information [31-36]. Other studies employed an interactive approach to educational meetings, including case study discussions, hands-on exercises in small teams, and human patient simulation scenarios [37-39]. The educational meetings ranged in duration and frequency-from single education sessions (i.e., 30-min training sessions) to more multi-phased interventions that included multiple educational meetings over time (i.e., one, 2-h meeting per month for 6 months) [40].

A majority of studies $(n=23 / 41)$ used implementation strategies not included in the EPOC taxonomy (Table 5), which consisted of the following: adaptation of practice guidelines to local context $(n=9)$, external facilitation $(n=14)$, and changes to organizational policy $(n=3)$. In addition, a sample of the included studies also reported details of the development and delivery of specific 
Table 2 Risk of bias of included studies

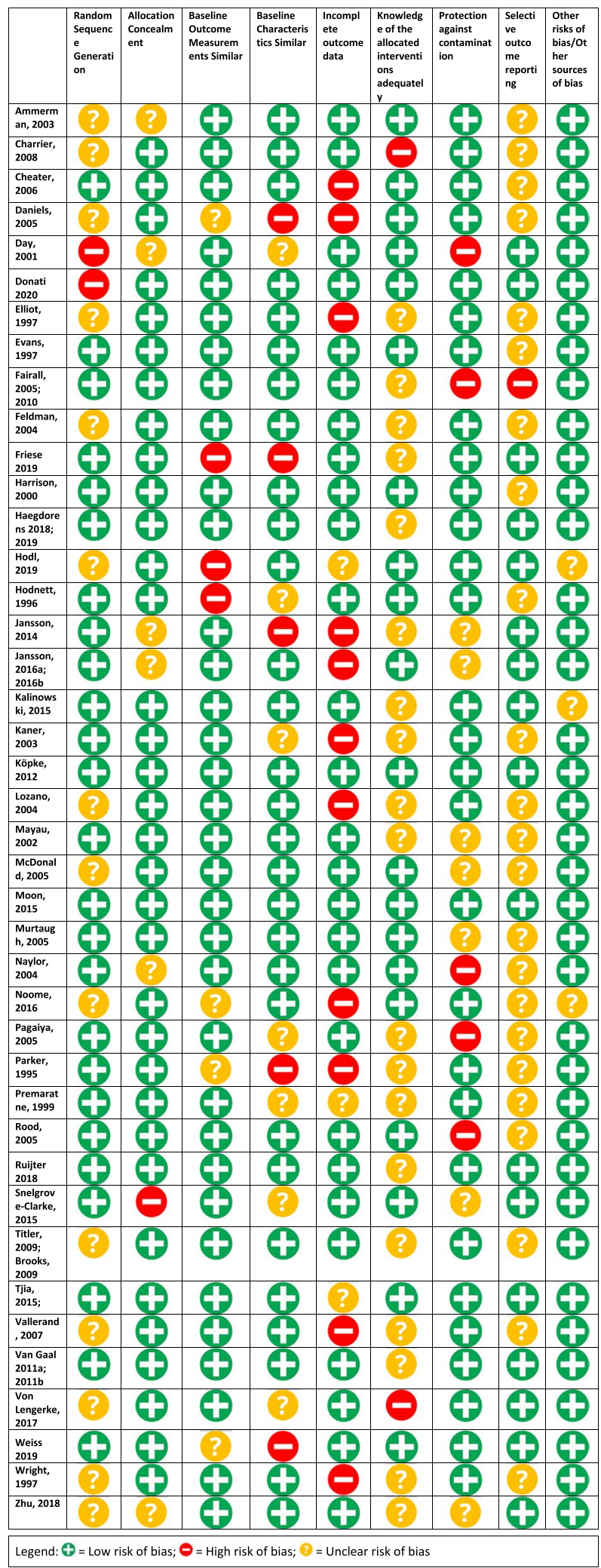

implementation strategies, indicating participatory cocreation approach during implementation ( $n=3$ studies). Table 5 outlines these four additional implementation strategies and compares them to the EPOC taxonomy.

\section{Implementation strategy effects}

One hundred and two outcomes were measured across the 41 studies. The most common outcomes were professional practice $(n=49)$, followed by patient health status $(n=26)$, professional knowledge $(n=14)$, expenditure $(n=8)$, resource use $(n=5)$. The majority of outcomes $(60 \%)$ were reported as positive and significant, including $64 \%(n=9 / 14)$ of professional knowledge outcomes, $59 \%(n=29 / 49)$ of professional practice outcomes, $54 \%$ $(n=14 / 26)$ patient health status outcomes, $80 \%(n=4 / 5)$ of resource use outcomes, and $63 \%(n=5 / 8)$ of expenditure outcomes. A summary of study outcomes is reported in Table 3.

We grouped the implementation strategies into five mutually exclusive categories to provide a narrative synthesis of study results. We created a sixth non-mutually exclusive category to describe multi-component strategies that were composed implementation strategies not included in EPOC taxonomy.

\section{Educational meetings alone}

Seven studies evaluated educational meetings alone [35, $39,40,51-54]$. Five studies reported positive and significant effects on professional practice outcomes [39, 40, 51, $53,54]$ and one study reported no effect [51]. Two studies reported positive and significant effects on professional knowledge $[51,52]$ and two studies reported no effects on patient health status outcomes $[35,39]$.

\section{Distribution of educational materials and educational meetings plus other interventions}

Twenty studies involved distribution of educational materials and educational meetings [31-34, 36, 55-69]. Of these studies, 10 examined professional practice outcomes, 11 examined patient health status outcomes, three examined professional knowledge, and one study examined resource use and expenditures (Table 3). Thirteen studies [32, 33, 36, 55-61, 63-65, 67] reported positive outcomes $(n=13)$ in all five outcome categories. All positive outcomes reported were statistically significant except two $[58,65]$. Mixed effects were reported on the remaining ten outcomes.

\section{Distribution of educational materials plus other interventions except educational meetings}

Eight studies involved distribution of educational materials plus other interventions except educational meetings [70-78]. Of these studies, two examined professional 


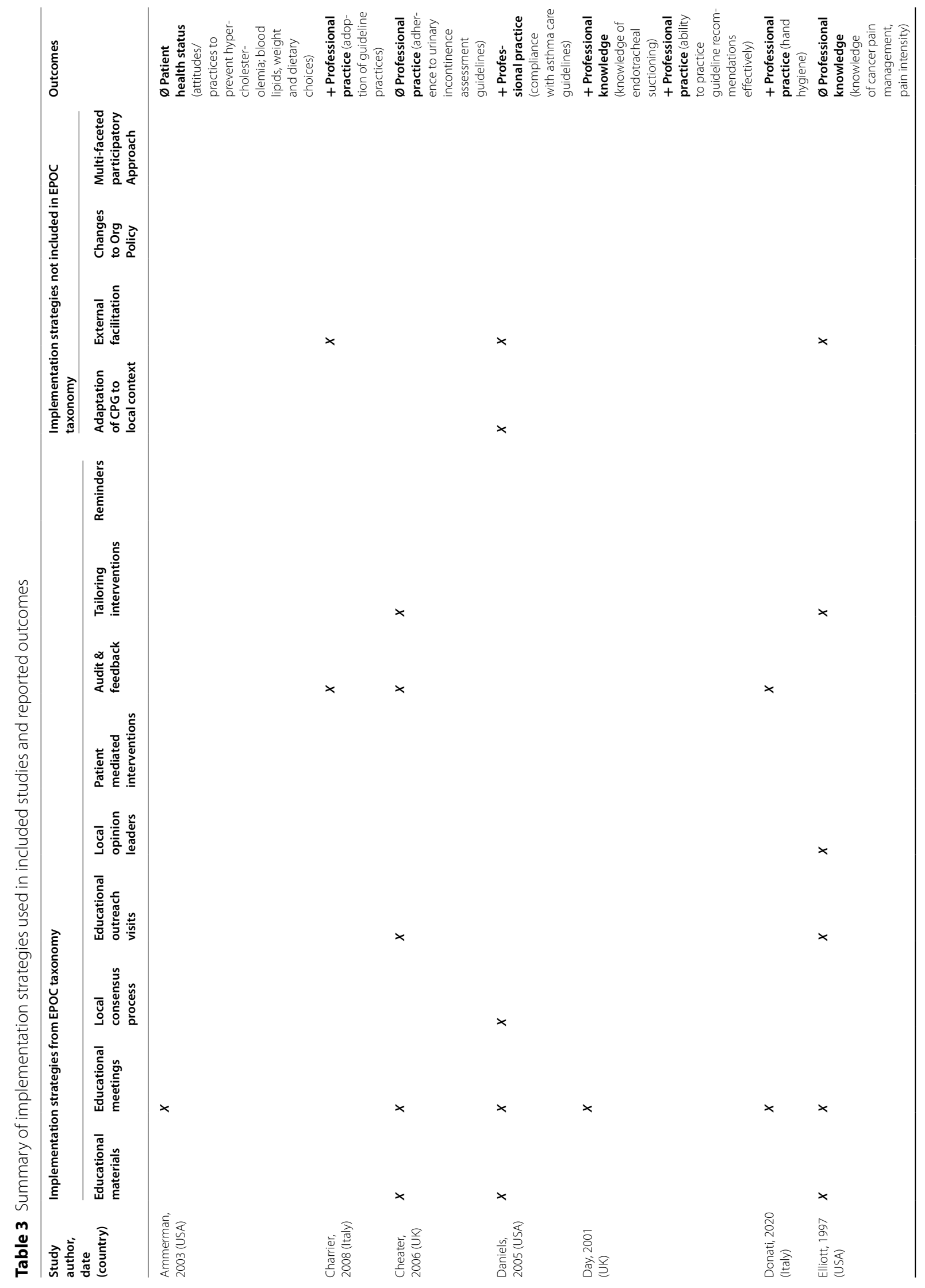




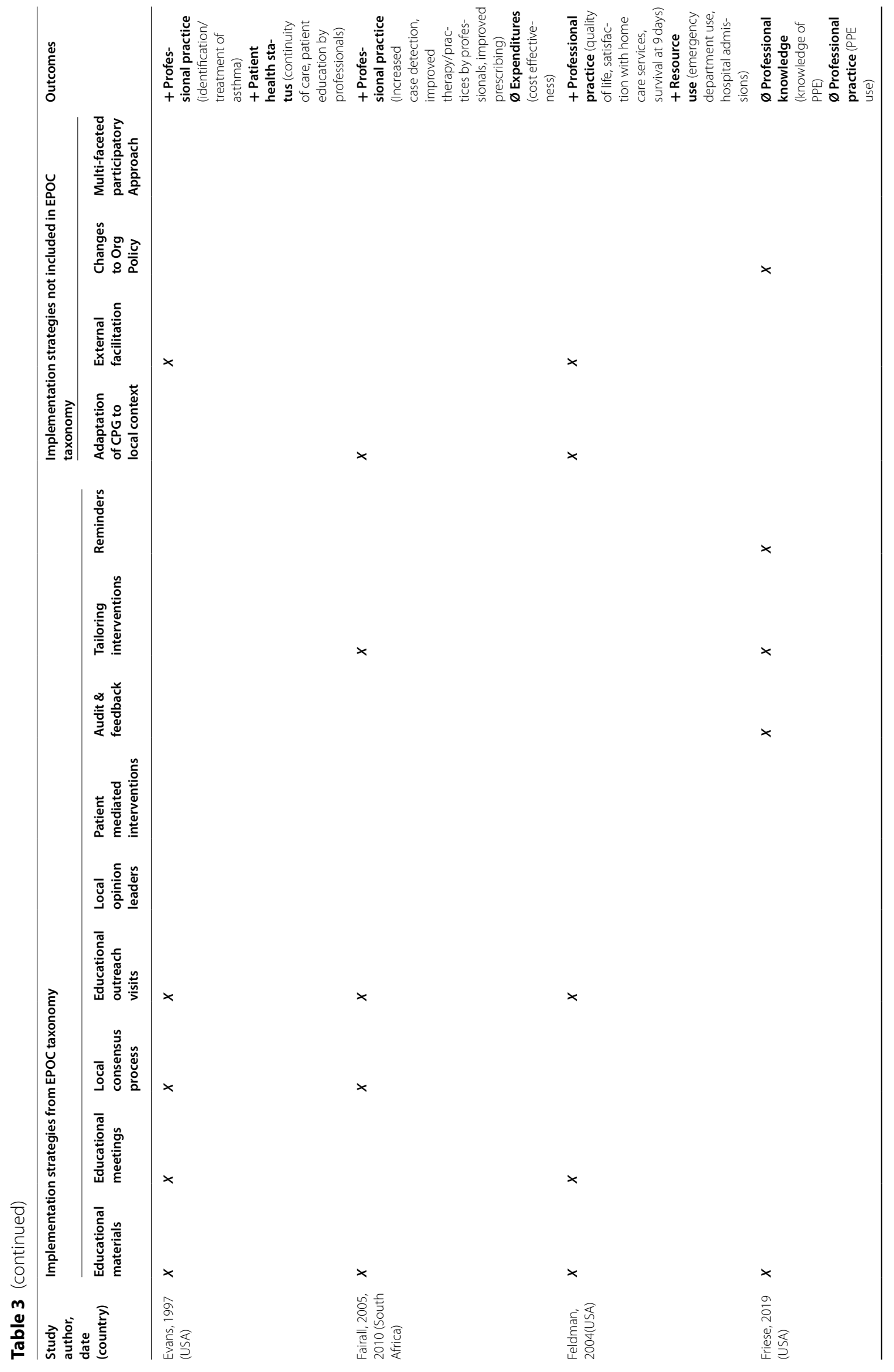




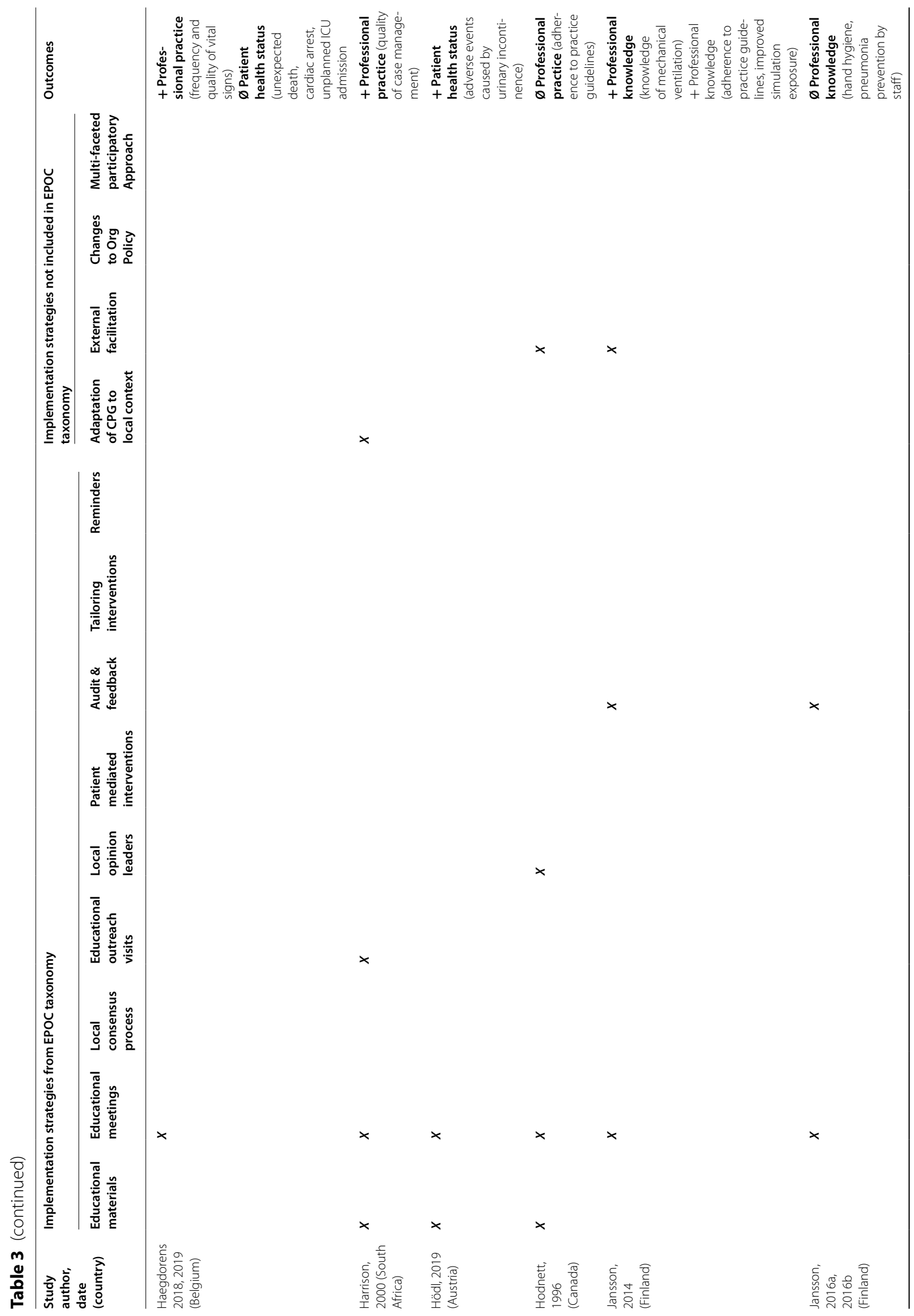




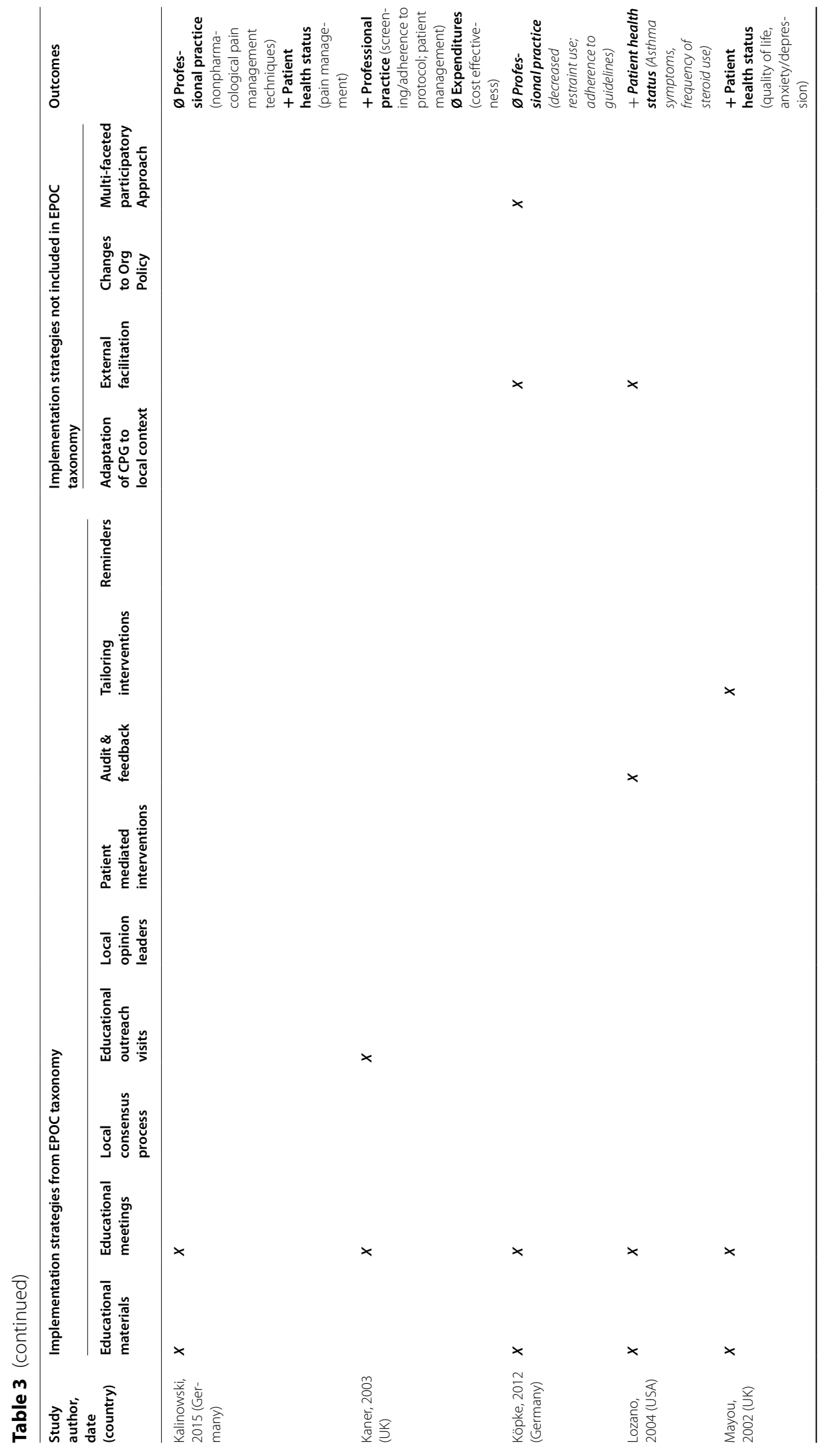




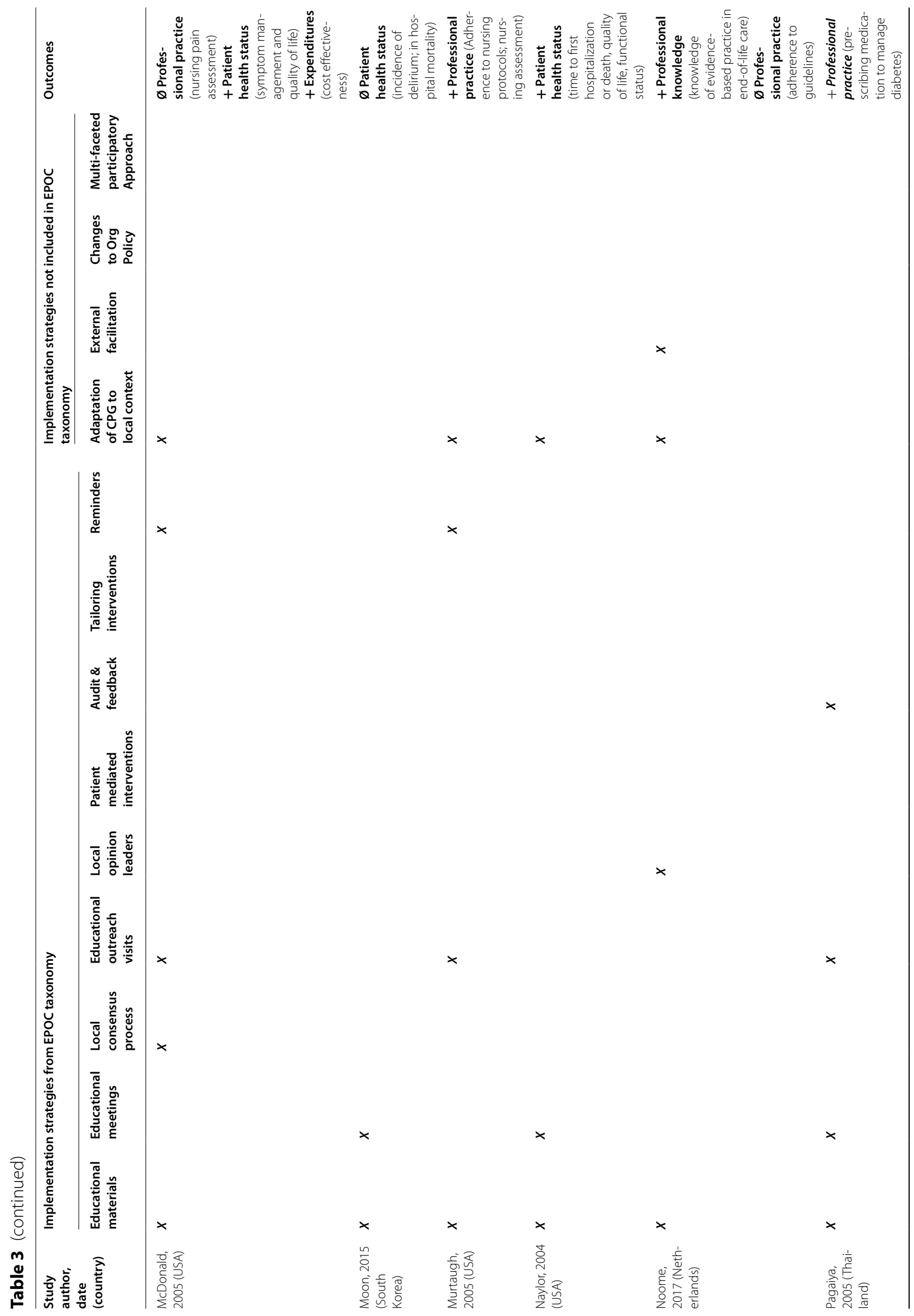




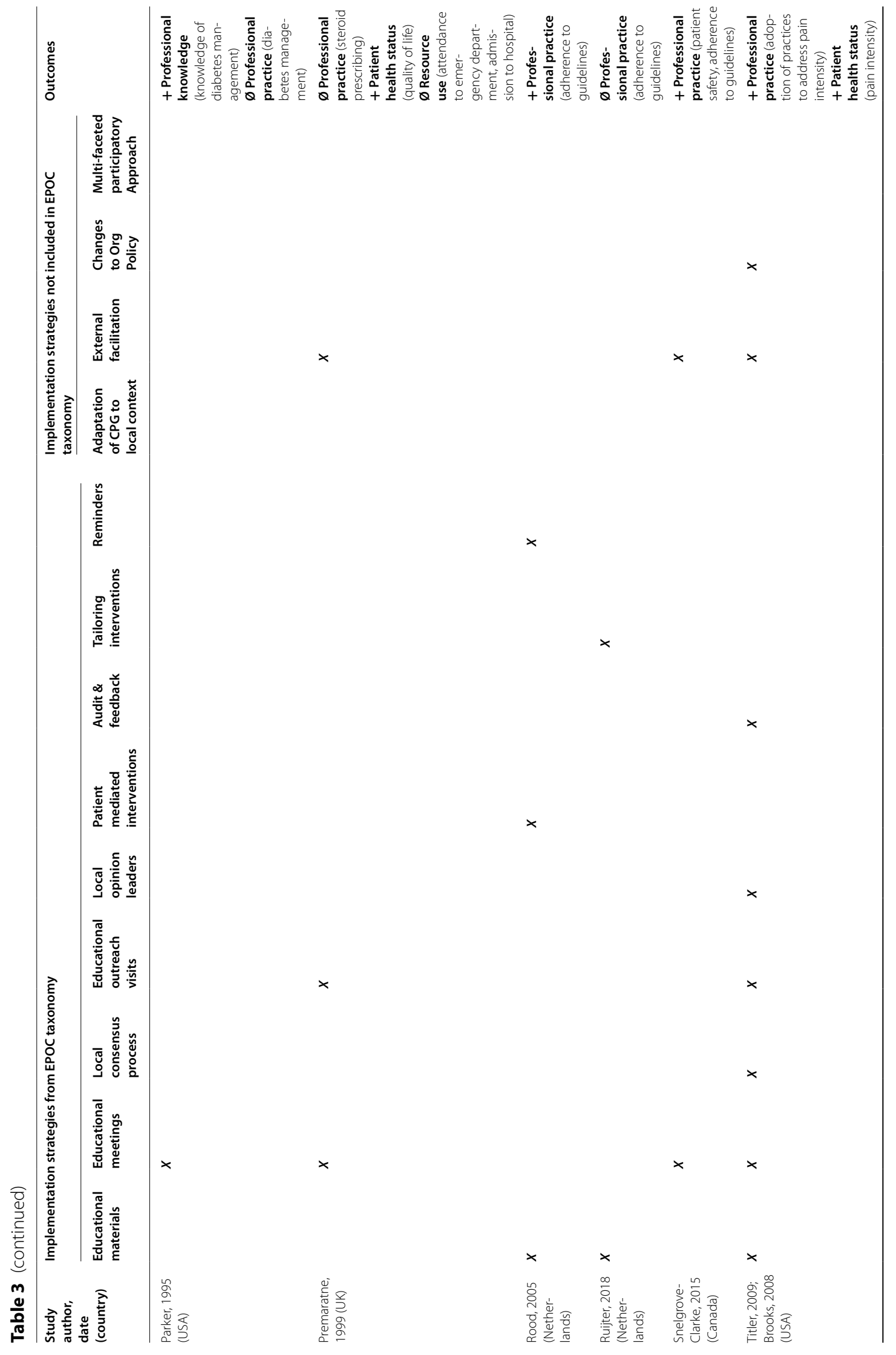




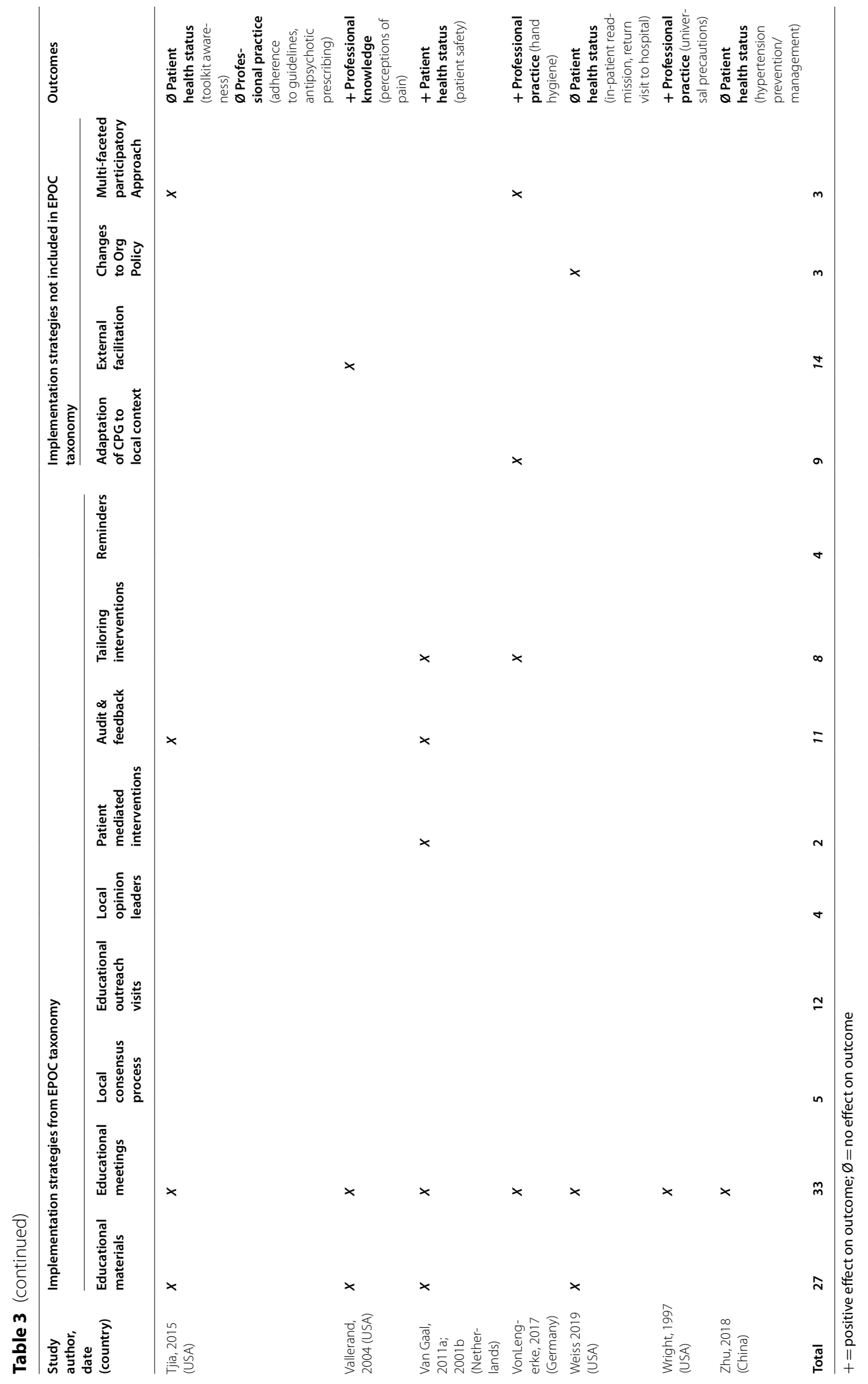


Table 4 Characteristics of reported educational strategies

\begin{tabular}{|c|c|c|c|}
\hline Author & Mode of delivery for educational strategy & Duration & Frequency \\
\hline Ammerman 2003 & Training session & $2 \mathrm{~h}$ per session & Once \\
\hline Cheater 2006 & $\begin{array}{l}\text { Lectures and discussions, video presentations, } \\
\text { observed role play, individual and peer feedback + } \\
\text { Written material provided and self-study }\end{array}$ & $1 / 2$ day & Twice \\
\hline Daniels 2005 & $\begin{array}{l}\text { Interactive case study discussions; hands on exer- } \\
\text { cises in small teams in the development of action } \\
\text { plans for patient self-monitoring and self-manage- } \\
\text { ment }+ \\
\text { Small groups also discussed effective ways to com- } \\
\text { municate specific messages to different audiences }\end{array}$ & NR & NR \\
\hline Day 1991 & $\begin{array}{l}\text { Teaching program with didactic and interactive } \\
\text { approaches }+ \\
\text { Practical beside demonstrations }\end{array}$ & $2 \mathrm{~h}$ & Once \\
\hline Donati 2020 & $\begin{array}{l}\text { Interactive training }+ \\
\text { Observational data collected and discussed }\end{array}$ & $3 \mathrm{~h}+30 \mathrm{~min}$ & Once + every 3 months \\
\hline Elliott 1997 & $\begin{array}{l}\text { Educational session with lectures, small group } \\
\text { discussions, case studies and practicums }\end{array}$ & Full day & Twice \\
\hline Evans 1997 & $\begin{array}{l}\text { Teaching sessions }+ \\
\text { Monthly visits to clinics by a full-time nurse educator }\end{array}$ & $3 \mathrm{~h}$ & Once \\
\hline Fairrall 2005, 2010 & Educational outreach sessions & $1-3 h$ & $2-6$ sessions \\
\hline Feldman 2004 & $\begin{array}{l}\text { Interactive practitioner training utilized experience } \\
\text { facilitators, as well as role-playing and audiotaping }\end{array}$ & NR & NR \\
\hline Friese 2019 & $\begin{array}{l}\text { E-learning modules and quiz }+ \\
\text { Email reminders reinforcing content }+ \\
\text { Tailored videos based on baseline surveys }\end{array}$ & NR & Quarterly \\
\hline Haegdorens 2018, 2019 & $\begin{array}{l}\text { Interactive training session led by experienced } \\
\text { practicing nurses }\end{array}$ & $4 \mathrm{~h}$ & Once \\
\hline Harrison, 2000 & $\begin{array}{l}\text { Training program with participation of one senior } \\
\text { primary healthcare nurse from each intervention } \\
\text { clinic. The workshop provided detailedinformation } \\
\text { about guidelines. Participants used aproblem-solv- } \\
\text { ing exercise to define objectives to improve quality } \\
\text { of STD managementin their clinics, which they then } \\
\text { carried out. + } \\
\text { Follow-up sessions were held in each clinic, address- } \\
\text { ing the topics of physicalexamination and history } \\
\text { taking, counseling and attitudes, and feedback of } \\
\text { STDsurveillance results + } \\
\text { A member of the district STD team made monthly } \\
\text { follow-up visitsto each clinic to provide regular } \\
\text { contact, and answer questions about the syndrome- } \\
\text { packets or other aspects of the training. }\end{array}$ & $\begin{array}{l}\text { Full-day } \\
\text { NR } \\
\text { NR }\end{array}$ & $\begin{array}{l}\text { Twice } \\
3 \\
\text { Monthly }\end{array}$ \\
\hline Hodl 2019 & $\begin{array}{l}\text { Instructional meeting }+ \\
\text { Recommendations and supplementary documents } \\
\text { (both hardcopy and PDF formats) }\end{array}$ & $1 \mathrm{~h}$ & Once \\
\hline Hodnett 1996 & $\begin{array}{l}\text { Workshop including lectures, panel discussions, role } \\
\text { playing, small group discussions and audio-visual } \\
\text { exhibits }\end{array}$ & NR & NR \\
\hline Jansson 2014 & $\begin{array}{l}\text { Human patient simulation (HPS) education with } \\
\text { scenario }+ \\
\text { Verbal feedback }+ \\
\text { Structured debriefing }\end{array}$ & $\begin{array}{l}20 \text { min with } 10-\text { min scenario } \\
60 \text {-min structured debriefing }\end{array}$ & Once \\
\hline Jansson 2016a, 2016b & $\begin{array}{l}\text { Human patient simulation (HPS) education with } \\
\text { scenario + verbal feedback + structured debriefing }\end{array}$ & $\begin{array}{l}20 \text { min with } 10-\text { min scenario } \\
60-\text { min structured debriefing }\end{array}$ & Once \\
\hline Kalinowksi 2015 & $\begin{array}{l}\text { Education program (seminar with oral presentations, } \\
\text { exercises and discussions) }+ \\
\text { Printed short summary of the clinical practice } \\
\text { guideline }\end{array}$ & $6 \mathrm{~h}$ & once \\
\hline
\end{tabular}


Table 4 (continued)

\begin{tabular}{|c|c|c|c|}
\hline Author & Mode of delivery for educational strategy & Duration & Frequency \\
\hline Kaner 2003 & $\begin{array}{l}\text { During outreach visit to the practice, nurses received } \\
\text { the screening and brief alcohol intervention (SBI) } \\
\text { program plus training on how to use the program. } \\
\text { Two weekly telephone calls which provided support } \\
\text { and advice about SBI. }\end{array}$ & Mean duration: $34 \mathrm{~min}$ & Once \\
\hline Kopke 2012 & $\begin{array}{l}\text { Structured education program for all nursing staff }+ \\
\text { External structured intensive training workshop for } \\
\text { nominated key nurses from different nursing homes } \\
+ \\
\text { Printed supportive material (guideline's 16-page } \\
\text { short version, flyer for relatives, posters) }\end{array}$ & Intensive training workshop 1 day & Once \\
\hline Lazono 2004 & $\begin{array}{l}\text { Workshops + } \\
\text { Central support by an educational coordinator + } \\
\text { An ongoing network for peer leaders via national } \\
\text { and local teleconferences + } \\
\text { Each leader received a tool kit containing the guide- } \\
\text { lines, key targets for behavior change, supporting } \\
\text { reference articles, laminated pocket cards summariz- } \\
\text { ing the approach to diagnosis and treatment, and } \\
\text { academic } \\
\text { detailing sheets on prescribing, trigger control and } \\
\text { specialty referral + } \\
\text { A tool kit of patient educational materials was also } \\
\text { provided to each practice + } \\
\text { The educational coordinator attempted to contact } \\
\text { each leader every } 1 \text { to } 2 \text { months to provide ideas, } \\
\text { materials and support; identify and resolve barriers } \\
\text { to change; and encourage less active leaders. }\end{array}$ & NR & Two workshops \\
\hline Mayou 2002 & $\begin{array}{l}\text { Trained and supervised by the researchers }+ \\
\text { Treatment was specified in a handbook }\end{array}$ & NR & NR \\
\hline McDonald 2005 & $\begin{array}{l}\text { Information package via email with guideline details } \\
+ \\
\text { Outreach by a Clinical Nurse Specialist who served } \\
\text { as an "expert peer." Standard email message from } \\
\text { CNS one week after the first email and reminded the } \\
\text { nurse that the CNS was available for consultation }\end{array}$ & NR & $N R$ \\
\hline Moon 2015 & Training sessions and educational material & $30 \mathrm{~min}$ & 2 sessions \\
\hline Murtaugh 2005 & $\begin{array}{l}\text { Information package via email with guideline details } \\
+ \\
\text { Outreach by a Clinical Nurse Specialist who served } \\
\text { as an "expert peer". Standard email message from } \\
\text { CNS one week after the first email asking about } \\
\text { the status of the eligible patient, whether the HF } \\
\text { self-care guide was useful, and whether there was } \\
\text { a patient issue the nurse would like to discuss with } \\
\text { the CNS. }\end{array}$ & NR & NR \\
\hline Naylor 2004 & $\begin{array}{l}\text { Orientation and training program on guideline } \\
\text { content }\end{array}$ & 2 months & Once \\
\hline Noome 2016 & $\begin{array}{l}\text { Educational meetings for the implementation } \\
\text { leaders (two nurses in each ICU were chosen as the } \\
\text { implementation leaders) }\end{array}$ & 1 day & Twice over 9 months \\
\hline Pagaiya 2005 & $\begin{array}{l}\text { Workshop with lectures, group discussions, role play } \\
\text { and presentations }+ \\
\text { Educational outreach visit by nurse practitioners }\end{array}$ & 3 days & Once \\
\hline Parker 1995 & $\begin{array}{l}\text { Educational program of lecture format followed by a } \\
\text { question-and-answer period }\end{array}$ & 20-min sessions & 7 sessions conducted 2 weeks apart \\
\hline Premaratne 199 & $\begin{array}{l}\text { Nurse specialists provided teaching sessions on core } \\
\text { elements of asthma care to all practice nurses + } \\
\text { Outreach visits by the nurse specialists to help the } \\
\text { practice nurse organize the clinic in keeping with } \\
\text { their teaching, and assist them in improving the } \\
\text { management of their patients. }\end{array}$ & NR & 6 sessions \\
\hline
\end{tabular}


Table 4 (continued)

\begin{tabular}{|c|c|c|c|}
\hline Author & Mode of delivery for educational strategy & Duration & Frequency \\
\hline Rood 2005 & $\begin{array}{l}\text { Computer-based version of guideline - received } \\
\text { guideline information via the clinical information } \\
\text { system }+ \\
\text { Paper based-version of guideline, 4-page flow chart } \\
\text { that directs nurse to relevant guideline advise }\end{array}$ & NR & NR \\
\hline Rejuiter 2018 & $\begin{array}{l}\text { Computer based e-learning program + } \\
\text { Tailored advice }\end{array}$ & 6 months & NR \\
\hline Snelgrove-Clarke 2015 & $\begin{array}{l}\text { Educational meetings }+ \\
\text { Personalized feedback by individualized coaching }\end{array}$ & $\begin{array}{l}2 \mathrm{~h} \\
\mathrm{NR}\end{array}$ & $\begin{array}{l}\text { Monthly } \\
\text { Monthly }\end{array}$ \\
\hline Titler 2009; Brooks 2008 & $\begin{array}{l}\text { Continuing Education program for senior adminis- } \\
\text { trative leaders+ } \\
\text { Train the trainer program: education of nurse opin- } \\
\text { ion leaders and change champions }+ \\
\text { Education of nursing and medical staff via a web- } \\
\text { based course + } \\
\text { Advanced practice nurse outreach every } 3 \text { weeks as } \\
\text { consultant to nurses and physicians + } \\
\text { Teleconferences to discuss issues, strategies for } \\
\text { overcoming perceived } \\
\text { barriers, progress made in education of staff, and } \\
\text { revision of policies and } \\
\text { documentation forms }\end{array}$ & $\begin{array}{l}60 \text { min } \\
3 \text { days } \\
\text { NR } \\
\text { NR } \\
\text { NR }\end{array}$ & $\begin{array}{l}\text { Once } \\
\text { Once } \\
\text { NR } \\
\text { NR } \\
\text { Monthly }\end{array}$ \\
\hline Tjia 2015 & Mailed toolkit & $\mathrm{n} / \mathrm{a}$ & Once \\
\hline Vallerand 2004 & $\begin{array}{l}\text { Lecture and discussions }+ \\
\text { Packet of information }+ \\
\text { Role-playing and assertiveness training }+ \\
\text { Principal investigator (an expert consultant) was } \\
\text { available by pager to provide support to nurses }\end{array}$ & $4 \mathrm{~h}$ & Once \\
\hline van Gaal 2011a, 2011b & $\begin{array}{l}\text { Educational meeting }+ \\
\text { Case discussions on every ward }+ \\
\text { Educational materials via CD ROMs }\end{array}$ & $\begin{array}{l}1.5 \mathrm{~h} \\
30 \mathrm{~min}\end{array}$ & $\begin{array}{l}\text { Once } \\
\text { Twice }\end{array}$ \\
\hline von Lengerke 2017 & $\begin{array}{l}\text { Tailored educational training for nurses }+ \text { feedback } \\
\text { discussions (from clinical managers and head } \\
\text { nurses) }\end{array}$ & NR & NR \\
\hline Weiss 2019 & Mandatory training & NR & $N R$ \\
\hline Wright 1997 & $\begin{array}{l}\text { Computer assisted intervention that presented } \\
\text { several patient scenarios }\end{array}$ & NR & NR \\
\hline Zhu 2018 & $\begin{array}{l}\text { Training program study to enhance the nurses' } \\
\text { decision-making }\end{array}$ & $36 \mathrm{~h}$ & NR \\
\hline
\end{tabular}

knowledge, seven examined professional practice outcomes, one study examined patient health status outcomes and resource use, and three studies examined expenditures (Table 3). One study reported positive and significant outcomes in professional knowledge [77], four studies reported positive and significant outcomes in professional practice $[70,72,73,76]$, and varying effects were found on resource use and expenditure outcomes [70].

\section{Educational meetings and other interventions except distribution of education materials}

Five studies evaluated use of an educational meetings plus other interventions but did not distribute educational materials. One study reported professional knowledge outcomes, four examined professional practice outcomes, two examined patient health status outcomes, and one examined resource use. Of these studies, positive and significant effects were reported on one professional knowledge outcome [37], two professional practice outcomes $[37,79,80]$, and positive but nonsignificant effects were reported on one patient health status outcome [80]. Three studies reported varying effects on professional practice [38], patient health status outcomes [81], and resource use [80].

\section{Other interventions that did not include educational meetings or distribution of educational materials}

Only one study evaluated other implementation strategies that did not include educational meetings or educational materials. Charrier et al. [82] evaluated two 


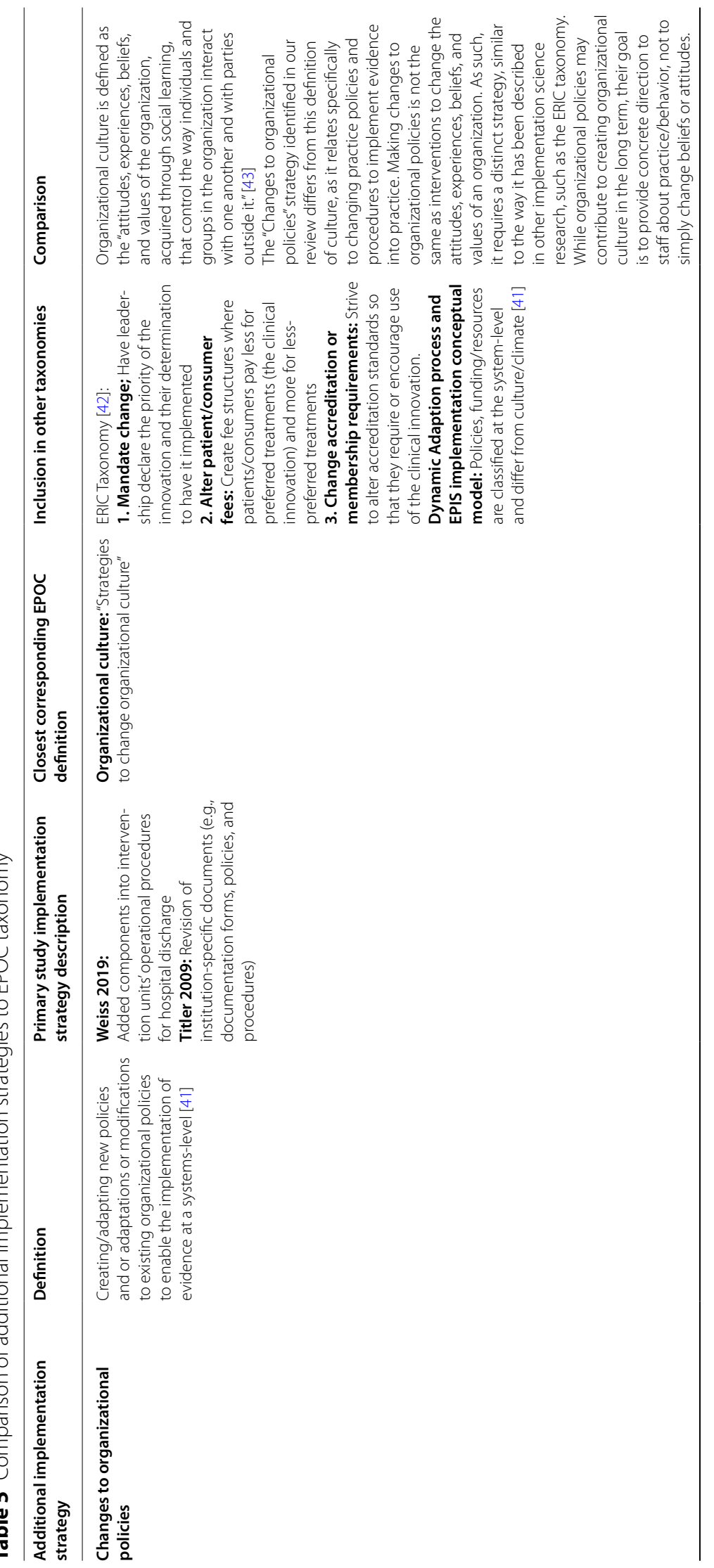




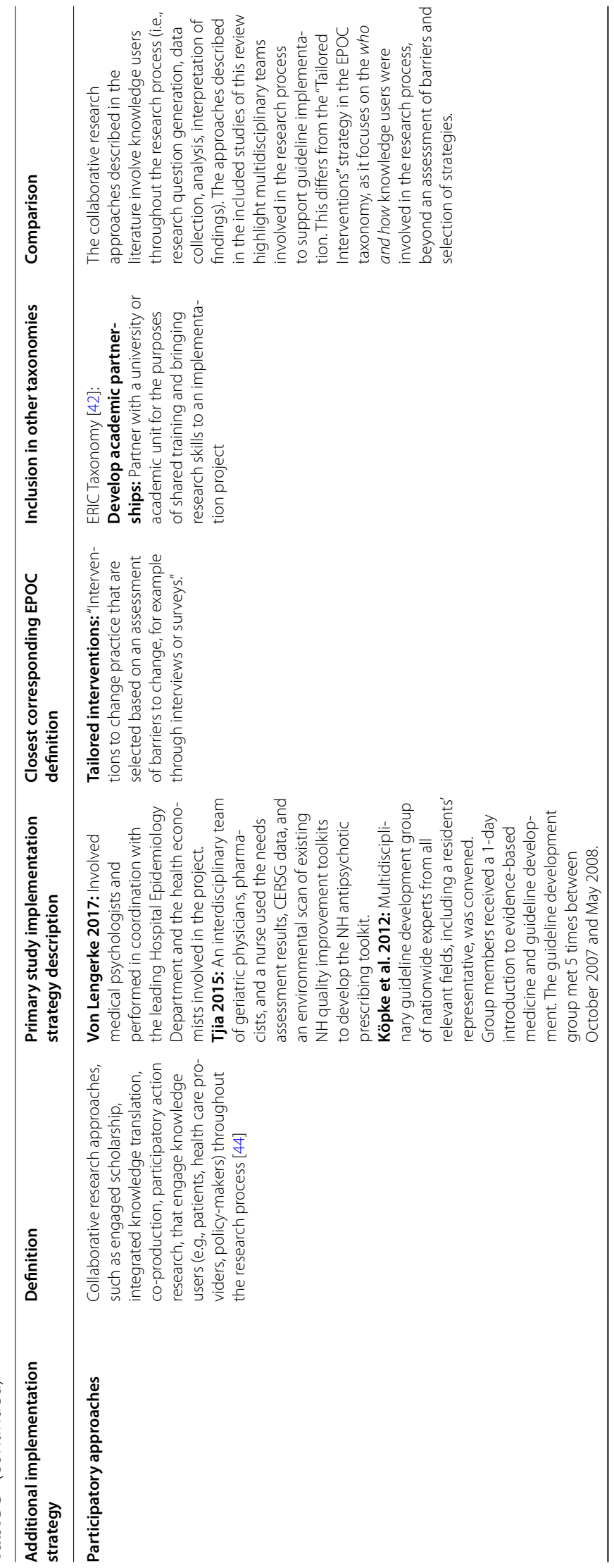




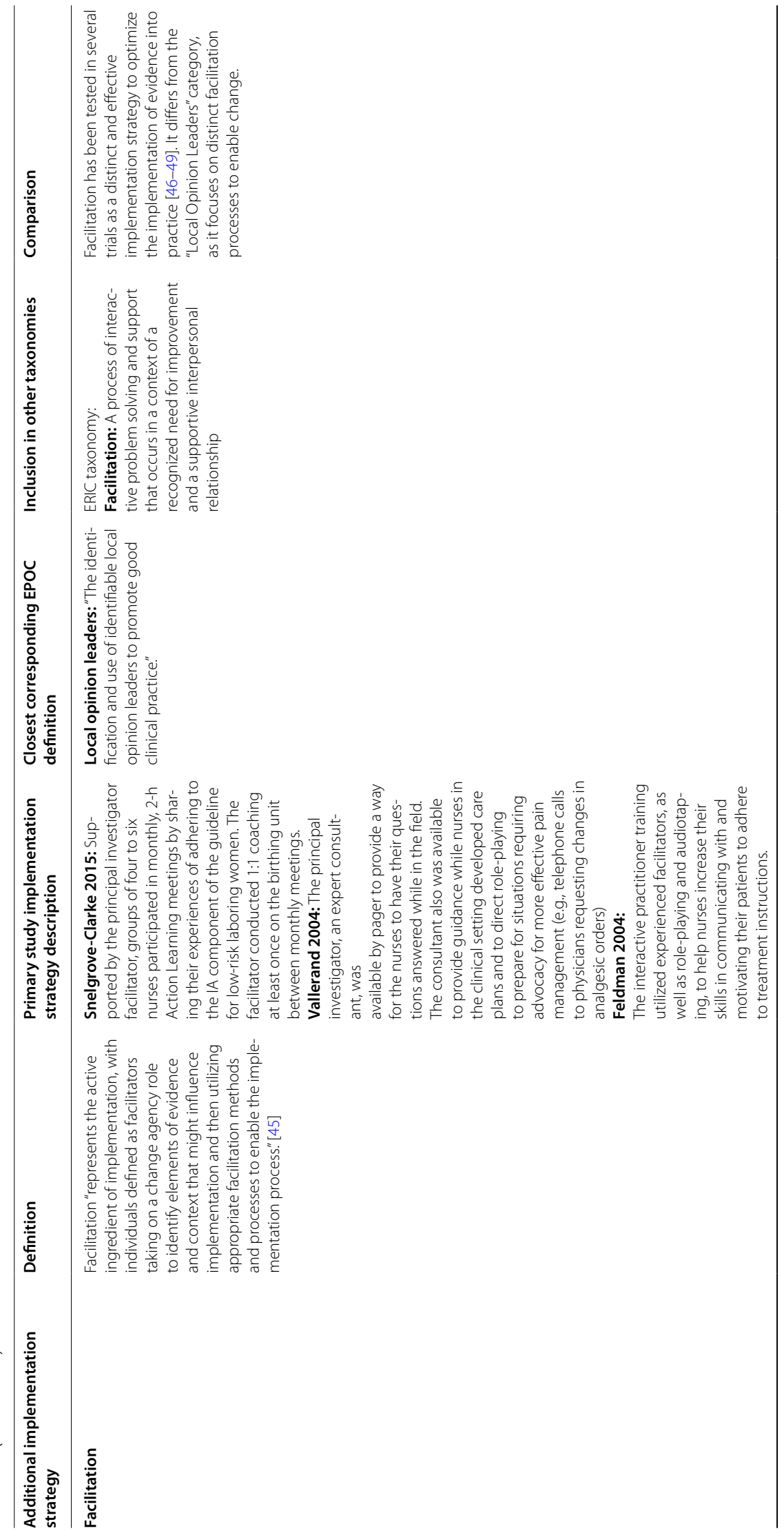




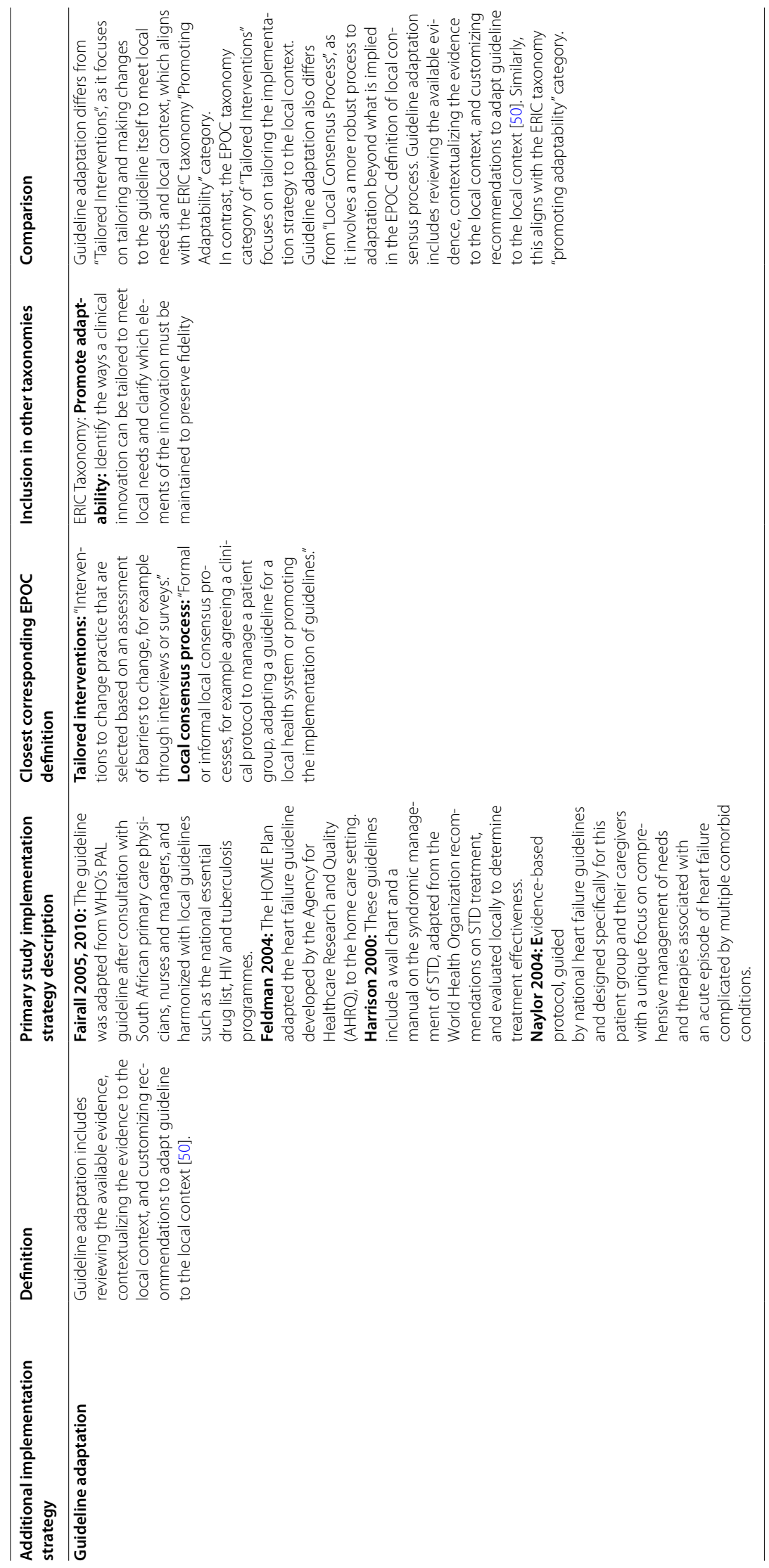


implementation strategies-audit and feedback and external facilitation and reported positive and significant effects on professional practice.

\section{Interventions not included in EPOC taxonomy and educational interventions}

Twenty-eight studies included a combination of educational strategies and other implementation strategies not included in EPOC taxonomy: adaptation of practice guidelines to local context $(n=9)$, external facilitation $(n=14)$, and changes to organizational policy $(n=3)$ (Table 3; Table 5). In addition, three studies also reported details of the development and delivery of specific implementation strategies, indicating participatory co-creation approach during implementation $(n=3)$ (Table 3; Table 5). Of these 23 studies, 26/43 outcomes were reported as positive $(n=26,60 \%)$. More specifically, four studies reported positive and significant effects on professional knowledge, ten studies reported positive and significant effects on professional practice, seven studies reported positive and significant effects on patient health status outcomes, and five studies reported positive outcomes on resource use and expenditure outcomes (Table 3).

\section{Discussion}

\section{Summary of evidence}

We synthesized the findings from 41 studies (reported in 46 papers) on guideline implementation strategies for nursing practice. Multi-component educational interventions were most commonly used and included a combination of educational materials, educational meetings, and educational outreach $(n=36)$. Studies evaluating single implementation strategies focused on educational meetings alone $(n=5)$ or audit and feedback (with no educational component) $(n=1)$. Outcomes pertained to professional knowledge, professional practice, patient health status outcomes and less frequently, health system outcomes (resource use and expenditures). Given the combination and permutations of implementation strategies and outcomes, we were limited in the comparisons we could analyze. Meta-analysis was not possible owing in part to the heterogeneity among studies, including differences in implementation strategy content, mode of delivery, duration, and frequency, as well as outcomes collected (Table 3; Table 4). An important finding was that $56 \%$ of studies employed implementation strategies that are not included in the EPOC taxonomy, including the use of external facilitation $(n=14)$, multifaceted participatory approaches $(n=3)$, adaptation of practice guidelines to local context $(n=9)$, and changes to organizational policy $(n=3)$ (Table 5$)$.

Our analysis suggests that educational meetings, in combination with other educational strategies (i.e., materials, outreach visits), are highly used in nursing and likely an effective implementation strategy for guideline implementation in nursing. Distribution of educational materials alone is effective but may not be sufficient to impact outcomes. The majority of studies $(n=40)$ evaluated educational interventions on professional knowledge outcomes $(n=14)$, professional practice outcomes $(n=48)$, patient health status outcomes $(n=26)$, and resource use/expenditure outcomes $(n=13)$. Overall, positive effects were found on the majority of professional practice outcomes $(n=29,59 \%)$, professional knowledge outcomes $(n=9,64 \%)$, patient health status outcomes $(n=14,54 \%)$, resource use outcomes $(n=4$, $80 \%)$, and expenditure outcomes $(n=5,63 \%)$. Multicomponent implementation strategies composed of interventions not included in the EPOC taxonomy (i.e., participatory approaches, facilitation, changes to organizational policies) also demonstrated positive trends on professional knowledge, professional practice, patient health status, resource use, and expenditure outcomes. Of the 43 outcomes measured with these participatorybased implementation strategies, 26 were reported as positive $(n=26,60 \%)$.

Our findings on effective educational implementation strategies are in line with previous reviews of guideline implementation strategies in medicine [22], pharmacy [16], rehabilitation [13], and physiotherapy [83]. A previous review of knowledge translation interventions for promoting evidence-informed decision-making among nurses found that almost all studies identified in their review included an educational component [11]. The primary focus on educational implementation strategies assumes that nurses and other health care providers do not implement guidelines because they do not have the appropriate knowledge (i.e., barrier to guideline use is lack of knowledge of the guideline). However, many behavioral determinants, including but not limited to, an individual or group of individual's motivation, practice context, and social influences affect the implementation process and outcomes [84]. A substantive body of implementation science research has identified modifiable behavioral determinants and/ or contextual mechanisms related to implementation in health care $[85,86]$. To move beyond educational implementation strategies in nursing, the field needs to be assessing barriers to guideline use, including the professional and organizational barriers to change, and 
use this assessment to tailor interventions to the identified barriers [87, 88].

Quality of the evidence.

The field of nursing is producing good quality trials. In this review, over two-thirds of studies $(n=29,70 \%)$ were of high quality (as indicated by protection against contamination) with a low risk of bias. Comparatively, a previous review of guideline implementation studies in medicine [22] found $54.5 \%$ to be of high quality and $4.5 \%$ low quality (RCT $n=110$ ). Despite the differences in the number of studies in medicine compared with nursing, the quality of the studies appears to be similar in both fields. Other reviews on similar topics among allied health professions and nursing report low methodological quality of the analyzed studies; however, these reviews included non-randomized control trials, quasi-experimental, and/or observational studies $[11,13,16]$.

Reporting continues to be an issue identified in this review, as was also identified in previous systematic reviews in medicine [22], allied health [13, 15, 16, 83], and nursing [19]. Study quality in the nursing implementation field revealed that $25 \%$ of the risk of bias indicators were rated as unclear. Many factors are considered when assessing the methodological quality of included RCT studies such as risk of contamination, concealment of allocation, blinded assessments of outcomes, baseline measures, and follow up of professionals. Unfortunately, the documentation on how these issues are managed in a particular study is often less than adequate, thereby making it difficult to ascertain if it is inadequate reporting or inadequate trial procedures. Reporting guidelines exist for trials and intervention description (EQUATOR Network [89]), including CONSORT statements [90] and the TIDiER guidelines [91]. While some studies in this review preceded release of reporting guidelines, only few provided adequate intervention description that aligns with reporting guidelines for interventions. It was difficult to discern intervention dose to support replicability. Future implementation intervention studies should use reporting guidelines to clearly articulate intervention components and strengthen the evidence base on guideline implementation in nursing.

\section{Implementation strategies in nursing}

This review highlights several key discoveries for others involved in implementation work in nursing. First, nursing appears to be advancing the evidence base on implementation strategies. There is an increasing number of RCTs in recent years and over half $(n=23)$ of the studies included guideline implementation strategies not described in the EPOC taxonomy, including facilitation $(n=14)$, guideline adaptation to the local context $(n=9)$, changes to organizational policies $(n=3)$, and participatory approaches to research $(n=3)$ (Table 5). A growing body of evidence, stemming from the nursing literature, reports facilitation as an effective strategy to optimize the implementation of evidence into practice [46-48]. A systematic review of guideline implementation in primary care found that practices supported by facilitators were 2.76 times more likely to adopt evidence-based clinical practice guidelines [46]. Further, guideline adaptation to local context is an implementation strategy that relates to the planned action phases $[88,92,93]$ and highlight efforts to align an implementation strategy to the local context and build on existing knowledge locally about effective strategies to increase uptake of evidence-based practice [92]. Similarly, previous research has shown participatory research approaches, that focus on producing knowledge and implementing evidence that is relevant to the needs of knowledge users, is an important strategy to consider when implementing evidence into practice [44].

Many of the identified implementation strategies align with the existing implementation science frameworks. Leeman et al. [94] offer a five-component classification system for implementation strategies, including dissemination strategies, implementation process strategies, integration strategies, capacity-building strategies, and scale-up strategies. The more prevalent educational implementation strategies identified in this review align with the implementation process strategies category. Our additional strategies that are not included in the EPOC taxonomy (facilitation, participatory approach, adaptation of guidelines, and changes to organizational policies) align with the integration, capacity-building, and scale-up strategies. Further, the additional implementation strategies map onto the Consolidated Framework for Implementation Research (CFIR) framework [95] which illustrates how these strategies target known behavioral and contextual determinants, including the intervention characteristics, process, and inner setting domains. This differs from educational implementation strategies that target the characteristics of the individual (i.e., knowledge and beliefs about the intervention). By mapping the identified implementation strategies onto existing frameworks, it is clear that multi-component educational and participatory strategies are useful to address multiple stages of the implementation process, and target multilevel behavioral and contextual determinants of guideline implementation.

Identification of these additional implementation strategies is an important finding for nursing implementation research and practice and may help to move beyond traditional educational approaches to implementation. Facilitation, guideline adaptation, changes to 
organizational policy, and participatory approaches are strategies that target guideline implementation within teams, units, or organizations. In a nursing context of $24 / 7$ care, the decision-making process often occurs in a team or group context, as well as with individual practitioner decision-making $[18,96]$. This differs from previous reviews of implementation strategies in medicine, pharmacy, and dentistry where individual-based implementation strategies are singularly used (i.e., reminders, audit and feedback). These may not function in the same way within a team context. The concern is that by only categorizing implementation strategies similar to reviews with other health care providers, we may be missing an opportunity to understand how implementation works in nursing contexts. To this point, the majority of included nursing trials $(n=23 / 41)$ used implementation strategies not included in the EPOC review and showed positive impacts on patient, provider, and health system outcomes.

Overall, implementation researchers and practitioners may find results of our systematic review helpful moving forward. First, for those undertaking similar reviews in the future, there are other taxonomies that capture more of the strategies we identified through our inductive thematic analysis. Numerous taxonomies and classification schemes have been published that describe a range of implementation strategies [97]. For example, the Expert Recommendations for Implementing Change (ERIC) project provides a compilation of implementation strategies, including strategies such as facilitation, promote adaptability, assess for readiness, identify barriers and facilitators, and develop academic partnerships [42]. Further, Slaughter et al. 2017 [98] provide practical resources for implementation researchers that includes a variety of classification schemes for knowledge translation interventions. Nursing implementation researchers should aim to explore the effectiveness and feasibility of these additional types of interventions in future work. Second, nurses in practice settings or other disciplines that work primarily in teams may benefit from using a taxonomy that includes team-based implementation strategies to plan and execute their implementation projects.

A second important discovery is that pre-existing search strategies for implementation strategy literature in other health professions does not work for locating guideline implementation studies in the nursing literature. We duplicated the search strategies described in a previous review of implementation strategies used in medicine [28]; however, these strategies did not locate any relevant nursing literature. We then crafted our own extensive search strategy and located the papers included for this review. This may be related to the medicinefocused search strategy not including CINAHL or other nursing databases (e.g., Proquest Nursing and Allied Health or British Nursing Index) and not including all types of common nursing roles (e.g., registered nurses). As such, efforts are needed to go beyond pre-existing search strategies and taxonomies to capture nursing trials and the strategies employed. The nursing implementation science field could be advanced by a separate review of non-RCT studies to understand a broader base of implementation strategies. Findings from this present review highlight that the state of science is not mature enough for solely analyzing RCTs; useful information from other types of studies would supplement findings from RCTs. The current review provides the start of a search framework to be used and expanded on by others to explore additional guideline implementation strategies in nursing in quasi or non-experimental research designs and qualitative research studies.

\section{Future research directions}

A key finding from this review is the number of studies $(n=23)$ that used participatory-based implementation strategies (Table 3; Table 5). Unfortunately, due to heterogeneity of implementation strategies, direct comparisons between the 13 studies that only included EPOC taxonomy implementation strategies versus these 23 studies is not possible. Future research is needed to explore the effectiveness of these types of implementation strategies not included in the EPOC taxonomy. This will help to move this field beyond educational interventions and understand how different components of these strategies work in a nursing context and with what effect. Further, it will help practitioners select the most appropriate, feasible, and effective implementation strategies for their specific nursing context. As previously discussed, we recommend examining these implementation strategies in the context of descriptive and qualitative studies to understand what works, for whom, and in what context.

Importantly, the implementation science literature generally recommends the use of theory to guide intervention design and implementation. Use of theory supports development of interventions that target behavioral determinants and lead to potentially stronger effects [84, 99]. Further, theory use leads to evaluations that are more robust in developing a theoretical understanding of intervention effects [84]. Despite its benefits, to date, reviews in medicine and other allied health professions have not reported extensive use of theory in intervention design. In a review of guideline dissemination and implementation strategies in the cancer care context, only one of 33 included studies used theory to directly inform the design of the intervention [100]. Similarly, only 14 out of 158 studies included in a review of uptake of evidence-based 
interventions in maternity care reported the use of theory [101]. Notably, in the current review, $40 \%$ of studies used theory to inform the design of interventions including studies. It appears that nurses involved in implementation research are early adopters, with the use of theory in intervention design dating back to included studies published in 1997 [56]. This may be explained by the strong theoretical underpinning in nursing and the use of theory to inform nursing practice [102]. Regardless, this warrants further investigation to understand how theory is being used and what is its effect on implementation strategy development and outcomes in nursing.

\section{Limitations}

The following limitations of this systematic review should be considered. First, we used a broad definition of guidelines so our interpretation may differ from others. Second, only studies published in English were included and potentially relevant studies published in other languages may have been missed. Third, many papers lacked detail on the implementation strategies used, which made it challenging to synthesize similar strategies and understand the duration and frequency needed to have the desired effect. Lastly, we had hoped to be able to do a meta-analysis; however, a narrative review was conducted because the methodological and clinical heterogeneity of the studies in this review revealed that meta-analysis was not appropriate. This level of heterogeneity among implementation studies has also been found in similar reviews $[16,100]$ of implementation strategies and speaks to the need for further work in the field to understand implementation effectiveness and improve reporting.

\section{Conclusions}

In this review, we aimed to describe the use and effects of implementation strategies to facilitate the uptake of guidelines focused on nursing care. While the evidence is limited, multi-component educational strategies were most commonly tested. Implementation strategies that include educational strategies reported promising effects on professional knowledge, professional practice, patient health status, and resource use/expenditure outcomes. We discovered additional implementation strategies in these studies that are not currently included in the EPOC taxonomy. Strategies such as facilitation, guideline adaptation, using a participatory approach, and changing organizational policies may be useful for both nurses and others similarly working in teams in health care to implement practice guidelines. Future implementation research in nursing, using qualitative, quantitative, and mixed methods research designs, is needed to understand what works, for whom and in what context. This includes assessing barriers to guideline use and tailoring implementation strategies to identified barriers. Ultimately, these research efforts will help to strengthen the evidence on effective guideline implementation strategies in nursing practice. While not the purpose of our review, we have determined that implementation of guidelines by nurses, on balance, tends to improve the process of care, patient health outcomes and health system outcomes (resource use and costs). Hence, the need to continue studying how to effectively encourage adoption of guidelines in nursing is vital.

\section{Abbreviations}

RCT: Randomized controlled trials; CRCT: Cluster randomized controlled trials; APN: Advanced practice nurse; NP: Nurse practitioner; CE: Clinical educators; CNS: Clinical nurse specialist; LPN: Licensed practical nurse; RPN: Registered practical nurse; RN: Registered nurse; EPOC: Effective practice and organization of care.

\section{Supplementary Information}

The online version contains supplementary material available at https://doi. org/10.1186/s13012-021-01165-5.

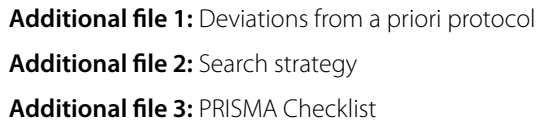

\section{Acknowledgements}

Not applicable.

\section{Authors' contributions}

$\mathrm{MBH}$ and IDG contributed to conception and design of the study. CG, PO, and ARW designed and ran the literature search. CG, CEC, VN, PK, and HG performed the screening, data extraction, and quality appraisal of included studies. CEC, MBH, CG, and IDG contributed to data interpretation and wrote the first draft of the manuscript. All authors critically reviewed and revised the manuscript and approved the final version.

\section{Funding}

This study was supported by the Integrated Knowledge Translation Research Network (CIHR FDN \#143237, PI: ID Graham).

Availability of data and materials

All data generated or analyzed during this study are included in this published article [and its supplementary information files]

\section{Declarations}

Ethics approval and consent to participate Not applicable.

Consent for publication

Not applicable.

Competing interests

The authors declare no competing interests.

\section{Author details}

${ }^{1}$ School of Nursing, Dalhousie University, 5860 University Ave., Halifax, NS B3H 4R2, Canada. 'School of Nursing, Queen's University, 92 Barrie Street, Kingston, ON K7L 3J9, Canada. ${ }^{3}$ Li Ka Shing Knowledge Institute of St. Michael's Hospital, 209 Victoria Street, Toronto, ON M5B 1W8, Canada. ${ }^{4}$ National Research Council 
Canada, Institute for Information Technology, 46 Dineen Drive, Fredericton, NB E3B 9W4, Canada. ${ }^{5}$ Queen's University Library, Queen's University, 18 Stuart Street, Kingston, ON K7L 3N6, Canada. ${ }^{6}$ School of Epidemiology and Public HealthSchool of Nursing, University of Ottawa, Ottawa Hospital Research Institute, 501 Smyth Road, Ottawa, ON K1H 8L6, Canada.

Received: 19 May 2021 Accepted: 12 October 2021 Published online: 04 December 2021

\section{References}

1. Straus S, Tetroe J, Graham ID. Knowledge translation in health care: moving from evidence to practice: Wiley; 2013. p. 359.

2. Grimshaw JM, Russell IT. Effect of clinical guidelines on medical practice: a systematic review of rigorous evaluations. Lancet Lond Engl. 1993;342(8883):1317-22.

3. Braithwaite J, Glasziou P, Westbrook J. The three numbers you need to know about healthcare: the 60-30-10 challenge. BMC Med. 2020;18(1):102.

4. Mackey A, Bassendowski S. The history of evidence-based practice in nursing education and practice. J Prof Nurs Off J Am Assoc Coll Nurs. 2017 Feb;33(1):51-5.

5. Institute of Medicine. Clinical Practice Guidelines We Can Trust [Internet]. Graham R, Mancher M, Miller Wolman D, Greenfield S, Steinberg E, editors. Washington (DC): National Academies Press (US); 2011 [cited 2020 May 11]. Available from: http://www.ncbi.nlm.nih.gov/books/ NBK209539/

6. Gundersen L. The effect of clinical practice guidelines on variations in care. Ann Intern Med. 2000;133(4):317-8.

7. Grol R. Successes and failures in the implementation of evidence-based guidelines for clinical practice. Med Care. 2001;39(8 Suppl 2):||46-54.

8. Rycroft-Malone J, Burton CR, Wilkinson J, Harvey G, McCormack B, Baker $\mathrm{R}$, et al. Collective action for implementation: a realist evaluation of organisational collaboration in healthcare. Implement Sci. 2016;11(1):17

9. Sheldon TA, Cullum N, Dawson D, Lankshear A, Lowson K, Watt I, et al. What's the evidence that NICE guidance has been implemented? Results from a national evaluation using time series analysis, audit of patients' notes, and interviews. BMJ. 2004;329(7473):999.

10. Fischer F, Lange K, Klose K, Greiner W, Kraemer A. Barriers and strategies in guideline implementation - a scoping review. Healthcare. 2016;4(3):36.

11. Yost J, Ganann R, Thompson D, Aloweni F, Newman K, Hazzan A, et al. The effectiveness of knowledge translation interventions for promoting evidence-informed decision-making among nurses in tertiary care: a systematic review and meta-analysis. Implement Sci IS [Internet]. 2015 14 [cited 2019 Nov 21];10. Available from: https://www.ncbi.nlm.nih. gov/pmc/articles/PMC4499897/

12. Prior M, Guerin M, Grimmer-Somers K. The effectiveness of clinical guideline implementation strategies--a synthesis of systematic review findings. J Eval Clin Pract. 2008;14(5):888-97.

13. Jones CA, Roop SC, Pohar SL, Albrecht L, Scott SD. Translating knowledge in rehabilitation: systematic review. Phys Ther. 2015;95(4):663-77.

14. van der Meulen E. Participatory and action-oriented dissertations: the challenges and importance of community-engaged graduate research. Qual Rep. 2011;16(5):1291-303.

15. Villarosa AR, Maneze D, Ramjan LM, Srinivas R, Camilleri M, George A. The effectiveness of guideline implementation strategies in the dental setting: a systematic review. Implement Sci. 2019 Dec 17:14(1):106.

16. Watkins $\mathrm{K}$, Wood $\mathrm{H}$, Schneider CR, Clifford R. Effectiveness of implementation strategies for clinical guidelines to community pharmacy: a systematic review. Implement Sci IS [Internet]. 2015 Oct 29 [cited 2019 Dec 10];10. Available from: https://www.ncbi.nlm.nih.gov/pmc/articles/ PMC4627629/

17. Institute of Medicine. Clinical practice guidelines: directions for a new program [Internet]. Field MJ, Lohr KN, editors. Washington (DC): National Academies Press (US); 1990 [cited 2020 May 11]. Available from: http://www.ncbi.n/m.nih.gov/books/NBK235751/
18. Fernandez R, Johnson M, Tran DT, Miranda C. Models of care in nursing: a systematic review. Int J Evid Based Healthc. 2012;10(4):324-37.

19. Spoon D, Rietbergen T, Huis A, Heinen M, van Dijk M, van BodegomVos L, et al. Implementation strategies used to implement nursing guidelines in daily practice: a systematic review. Int J Nurs Stud. 2020;111:103748.

20. Ioannidis JPA. The mass production of redundant, misleading, and conflicted systematic reviews and meta-analyses. Milbank Q. 2016:94(3):485-514

21. Karunananthan S, Maxwell LJ, Welch V, Petkovic J, Pardo JP, Rader T, et al PROTOCOL: when and how to replicate systematic reviews. Campbell Syst Rev. 2020;16(2):e1087.

22. Grimshaw JM, Thomas RE, MacLennan G, Fraser C, Ramsay CR, Vale $L$, et al. Effectiveness and efficiency of guideline dissemination and implementation strategies. Health Technol Assess Winch Engl. 2004 Feb;8(6):iii-v 1-72.

23. Proctor EK, Powell BJ, McMillen JC. Implementation strategies: recommendations for specifying and reporting. Implement Sci. 2013 Dec 1;8(1):139.

24. Bick D, Graham ID. Evaluating the impact of implementing evidencebased practice. Vol. 1. John Wiley \& Sons; 2010.

25. Innovation $\mathrm{VH}$. Covidence systematic review software. Australia: Veritas Health Innovation Melbourne; 2016.

26. Hakkennes S, Dodd K. Guideline implementation in allied health professions: a systematic review of the literature. BMJ Qual Saf. 2008 Aug 1;17(4):296-300.

27. Cochrane Effective Practice and Organisation of Care. EPOC Taxonomy [Internet]. EPOC Taxonomy. 2015 [cited 2021 May 19]. Available from: / epoc-taxonomy

28. Hsieh H-F, Shannon SE. Three approaches to qualitative content analysis. Qual Health Res. 2005 Jan 11;15(9):1277-88.

29. Higgins JP, Altman DG. Assessing Risk of Bias in Included Studies. In: Cochrane Handbook for systematic reviews of interventions [Internet]. John Wiley \& Sons, Ltd; 2008 [cited 2020 May 11]. p. 187-241. Available from: https://onlinelibrary.wiley.com/doi/abs/10.1002/ 9780470712184.ch8

30. Popay J, Roberts H, Sowden A, Petticrew M, Arai L, Rodgers M, et al. Guidance on the conduct of narrative synthesis in systematic reviews. Prod ESRC Methods Programme Version. 2006;1:b92.

31. Cheater FM, Baker R, Reddish S, Spiers N, Wailoo A, Gillies C, et al. Cluster Randomized Controlled Trial of the Effectiveness of Audit and Feedback and Educational Outreach on Improving Nursing Practice and Patient Outcomes: Med Care 2006;44(6):542-551.

32. Hödl M, Halfens RJG, Lohrmann C. Effectiveness of conservative urinary incontinence management among female nursing home residents-a cluster RCT. Arch Gerontol Geriatr. 2019;81:245-51.

33. Kalinowski S, Budnick A, Kuhnert R, Könner F, Kissel-Kröll A, Kreutz R, et al. Nonpharmacologic pain management interventions in German nursing homes: a cluster randomized trial. Pain Manag Nurs. 2015;16(4):464-74.

34. Köpke S, Mühlhauser I, Gerlach A, Haut A, Haastert B, Möhler R, et al. Effect of a guideline-based multicomponent intervention on use of physical restraints in nursing homes: a randomized controlled trial. JAMA [Internet]. 201223 [cited 2020 May 5];307(20). Available from: http:// jama.jamanetwork.com/article.aspx?doi=10.1001/jama.2012.4517

35. Zhu X, Wong FKY, Wu CLH. Development and evaluation of a nurse-led hypertension management model: a randomized controlled trial. Int J Nurs Stud. 2018 Jan;77:171-8.

36. Weiss ME, Yakusheva O, Bobay KL, Costa L, Hughes RG, Nuccio S, et al. Effect of implementing discharge readiness assessment in adult medical-surgical units on 30-day return to hospital: the READI randomized clinical trial. JAMA Netw Open. 2019 04:2(1):e187387.

37. Jansson MM, Ala-Kokko TI, Ohtonen PP, Meriläinen MH, Syrjälä HP, Kyngäs HA. Human patient simulation education in the nursing management of patients requiring mechanical ventilation: a randomized, controlled trial. Am J Infect Control. 2014 Mar:42(3):271-6.

38. Jansson MM, Syrjälä HP, Ohtonen PP, Meriläinen MH, Kyngäs HA, AlaKokko TI. Randomized, controlled trial of the effectiveness of simulation education: a 24-month follow-up study in a clinical setting. Am J Infect Control. 2016:44(4):387-93. 
39. Haegdorens F, Van Bogaert P, Roelant E, De Meester K, Misselyn M, Wouters $\mathrm{K}$, et al. The introduction of a rapid response system in acute hospitals: a pragmatic stepped wedge cluster randomised controlled trial. Resuscitation. 2018;129:127-34.

40. Snelgrove-Clarke E, Davies B, Flowerdew G, Young D. Implementing a fetal health surveillance guideline in clinical practice: a pragmatic randomized controlled trial of action learning: fetal health surveillance guideline: an RCT of action leaning. Worldviews Evid-Based Nurs. 2015;12(5):281-8.

41. Aarons GA, Green AE, Palinkas LA, Self-Brown S, Whitaker DJ, Lutzker JR, et al. Dynamic adaptation process to implement an evidence-based child maltreatment intervention. Implement Sci. 2012;7(1):32.

42. Powell BJ, Waltz TJ, Chinman MJ, Damschroder LJ, Smith JL, Matthieu $\mathrm{MM}$, et al. A refined compilation of implementation strategies: results from the expert recommendations for implementing change (ERIC) project. Implement Sci. 2015;10(1):21.

43. Serrat O. A primer on organizational culture. In: Serrat O, editor. Knowledge solutions: tools, methods, and approaches to drive organizational performance [Internet]. Singapore: springer; 2017 [cited 2021 Sep 20]. p. 355-8. Available from: https://doi.org/10.1007/978-981-100983-9_40.

44. Hoekstra F, Mrklas KJ, Khan M, McKay RC, Vis-Dunbar M, Sibley KM, et al. A review of reviews on principles, strategies, outcomes and impacts of research partnerships approaches: a first step in synthesising the research partnership literature. Health Res Policy Syst. 2020;18(1):51.

45. Harvey G, McCormack B, Kitson A, Lynch E, Titchen A. Designing and implementing two facilitation interventions within the 'facilitating implementation of research evidence (FIRE)'study: a qualitative analysis from an external facilitators' perspective. Implement Sci. 2018;13(1):141.

46. Baskerville NB, Liddy C, Hogg W. Systematic review and meta-analysis of practice facilitation within primary care settings. Ann Fam Med. 2012;10(1):63-74.

47. Dogherty EJ, Harrison M, Graham I, Keeping-Burke L. Examining the use of facilitation within guideline dissemination and implementation studies in nursing. Int J Evid Based Healthc. 2014;12(2):105-27.

48. Persson LÅ, Nga NT, Målqvist M, Thi Phuong Hoa D, Eriksson L, Wallin L, et al. Effect of facilitation of local maternal-and-newborn stakeholder groups on neonatal mortality: cluster-randomized controlled trial. PLoS Med. 2013;10(5):e1001445.

49. Seers K, Rycroft-Malone J, Cox K, Crichton N, Edwards RT, Eldh AC, et al. Facilitating Implementation of Research Evidence (FIRE): an international cluster randomised controlled trial to evaluate two models of facilitation informed by the Promoting Action on Research Implementation in Health Services (PARIHS) framework. Implement Sci IS [Internet]. 201816 [cited 2019 Apr 9];13. Available from: https://www. ncbi.nlm.nih.gov/pmc/articles/PMC6238407/

50. Harrison MB, Légaré F, Graham ID, Fervers B. Adapting clinical practice guidelines to local context and assessing barriers to their use. CMAJ Can Med Assoc J. 2010;182(2):E78-84.

51. Day T, Wainwright SP, Wilson-Barnett J. An evaluation of a teaching intervention to improve the practice of endotracheal suctioning in intensive care units. J Clin Nurs. 2001;10(5):682-96.

52. Parker MT, Leggett-Frazier N, Vincent PA, Swanson MS. The impact of an educational program on improving diabetes knowledge and changing behaviors of nurses in long-term care facilities. Diabetes Educ. 1995;21(6):541-5.

53. Lengerke T von, Lutze B, Krauth C, Lange K, Stahmeyer JT, Chaberny IF. Promoting hand hygiene compliance: PSYGIENE - a cluster-randomized controlled trial of tailored interventions. Dtsch Aerzteblatt Online [Internet]. 201720 [cited 2020 May 5]; Available from: https:// www.aerzteblatt.de/10.3238/arztebl.2017.0029

54. Wright BJ, Turner JG, Daffin P. Effectiveness of computer-assisted instruction in increasing the rate of universal precautions-related behaviors. Am J Infect Control. 1997;25(5):426-9.

55. Daniels E, Bacon J, Denisio S, Fry Y, Murray V, Quarshie A, et al. Translation squared: improving asthma care for high-disparity populations through a safety net practice-based research network. J Asthma. 2005;42(6):499-505.

56. Elliott TE, Murray DM, Oken MM, Johnson KM, Braun BL, Elliott BA, et al. Improving cancer pain management in communities: Main results from a randomized controlled trial. J Pain Symptom Manag. 1997;13(4):191-203.

57. Evans D, Mellins R, Lobach K, Ramos-Bonoan C, Pinkett-Heller M, Wiesemann S, et al. Improving care for minority children with asthma: professional education in public health clinics. PEDIATRICS. 1997;99(2):157-64.

58. Feldman PH, Peng TR, Murtaugh CM, Kelleher C, Donelson SM, McCann $\mathrm{ME}$, et al. A randomized intervention to improve heart failure outcomes in community-based home health care. Home Health Care Serv Q. 2004;23(1):1-23.

59. Harrison A, Karim SA, Floyd K, Lombard C, Lurie M, Ntuli N, et al. Syndrome packets and health worker training improve sexually transmitted disease case management in rural South Africa: randomized controlled trial: AIDS 2000;14(17):2769-2779.

60. Lozano P, Finkelstein JA, Carey VJ, Wagner EH, Inui TS, Fuhlbrigge AL, et al. A multisite randomized trial of the effects of physician education and organizational change in chronic-asthma care: health outcomes of the pediatric asthma care patient outcomes research team II study. Arch Pediatr Adolesc Med. 2004 Sep;158(9):875-83.

61. Mayou RA, Thompson DR, Clements A, Davies CH, Goodwin SJ, Normington $\mathrm{K}$, et al. Guideline-based early rehabilitation after myocardial infarction a pragmatic randomised controlled trial. J Psychosom Res. 2002;7.

62. Moon K-J, Lee S-M. The effects of a tailored intensive care unit delirium prevention protocol: a randomized controlled trial. Int J Nurs Stud. 2015;52(9):1423-32.

63. Naylor MD, Brooten DA, Campbell RL, Maislin G, McCauley KM, Schwartz JS. Transitional care of older adults hospitalized with heart failure: a randomized, controlled trial: follow-up of hospitalized elders. J Am Geriatr Soc. 2004;52(5):675-84.

64. Pagaiya N, Garner P. Primary care nurses using guidelines in Thailand: a randomized controlled trial. Tropical Med Int Health. 2005;10(5):471-7.

65. Titler MG, Herr K, Brooks JM, Xie X-J, Ardery G, Schilling ML, et al. Translating research into practice intervention improves management of acute pain in older hip fracture patients. Health Serv Res. 2009:44(1):264-87.

66. Tjia J, Field T, Mazor K, Lemay CA, Kanaan AO, Donovan JL, et al. Dissemination of evidence-based antipsychotic prescribing guidelines to nursing homes: a cluster randomized trial. J Am Geriatr Soc. 2015;63(7):1289-98

67. Hazard Vallerand A, Riley-Doucet C, Hasenau SM, Templin T. Improving cancer pain management by homecare nurses. Oncol Nurs Forum. 2004;31(4):809-16.

68. van Gaal BGI, Schoonhoven L, Mintjes JAJ, Borm GF, Hulscher MEJL, Defloor T, et al. Fewer adverse events as a result of the SAFE or SORRY? Programme in hospitals and nursing homes. Part I: primary outcome of a cluster randomised trial. Int J Nurs Stud. 2011;48(9):1040-8.

69. Hodnett ED, Kaufman K, O'Brien-Pallas L, Chipman M, Watson-MacDonell J, Hunsburger W. A strategy to promote research-based nursing care: Effects on childbirth outcomes. :8.

70. Fairall $L R$, Zwarenstein $M$, Bateman ED, Bachmann M, Lombard C, Majara BP, et al. Effect of educational outreach to nurses on tuberculosis case detection and primary care of respiratory illness: pragmatic cluster randomised controlled trial. Prim Care. 2005;331:5.

71. Friese CR, Yang J, Mendelsohn-Victor K, McCullagh M. Randomized Controlled Trial of an Intervention to Improve Nurses' Hazardous Drug Handling. Oncol Nurs Forum. 2019;46(2):248-56.

72. Kaner E, Lock C, Heather N, McNamee P, Bond S. Promoting brief alcohol intervention by nurses in primary care: a cluster randomised controlled trial. Patient Educ Couns. 2003;51(3):277-84.

73. Rood E. Use of a computerized guideline for glucose regulation in the intensive care unit improved both guideline adherence and glucose regulation. J Am Med Inform Assoc. 2004;12(2):172-80.

74. de Ruijter D, Candel M, Smit ES, de Vries H, Hoving C. The effectiveness of a computer-tailored E-learning program for practice nurses to improve their adherence to smoking cessation counseling guidelines: randomized controlled trial. J Med Internet Res. 2018;20(5):e193.

75. McDonald MV, Pezzin LE, Feldman PH, Murtaugh CM, Peng TR. Can just-in-time, evidence-based "reminders" improve pain management among home health care nurses and their patients? J Pain Symptom Manag. 2005;29(5):474-88. 
76. Murtaugh CM, Pezzin LE, McDonald MV, Feldman PH, Peng TR. Just-intime evidence-based E-mail "reminders" in home health care: impact on nurse practices: evidence-based E-mail reminders: nurse impact. Health Serv Res. 2005;40(3):849-64

77. Noome M, Dijkstra BM, van Leeuwen E, Vloet LCM. Effectiveness of supporting intensive care units on implementing the guideline'end-oflife care in the intensive care unit, nursing care': a cluster randomized controlled trial. J Adv Nurs. 2017;73(6):1339-54.

78. Fairall L, Bachmann MO, Zwarenstein M, Bateman ED, Niessen LW, Lombard C, et al. Cost-effectiveness of educational outreach to primary care nurses to increase tuberculosis case detection and improve respiratory care: economic evaluation alongside a randomised trial. Tropical Med Int Health. 2010;15(3):277-86.

79. Donati D, Miccoli GA, Cianfrocca C, Di Stasio E, De Marinis MG, Tartaglin D. Effectiveness of implementing link nurses and audits and feedback to improve nurses' compliance with standard precautions: a cluster randomized controlled trial. Am J Infect Control. 2020;48(10):1204-10.

80. Premaratne UN, Sterne JAC, Marks GB, Webb JR, Azima H, Burney PGJ. Clustered randomised trial of an intervention to improve the management of asthma: Greenwich asthma study. BMJ. 1999:318(7193):1251-5.

81. Ammerman AS, Keyserling TC, Atwood JR, Hosking JD, Zayed H, Krasny C. A randomized controlled trial of a public health nurse directed treatment program for rural patients with high blood cholesterols this work was supported in part by grant NR03042 from the National Center for nursing research, National Institutes of Health, Bethesda. Maryland Prev Med. 2003;36(3):340-51.

82. Charrier L, Allochis MC, Cavallo MR, Gregori D, Cavallo F, Zotti CM. Integrated audit as a means to implement unit protocols: a randomized and controlled study: audit for protocol implementation. J Eval Clin Pract. 2008;14(5):847-53.

83. van der Wees PJ, Jamtvedt G, RebbeckT, de Bie RA, Dekker J, Hendriks EJM. Multifaceted strategies may increase implementation of physiotherapy clinical guidelines: a systematic review. Aust J Physiother. 2008;54(4):233-41.

84. Michie S, Atkins L, West R. The behaviour change wheel (behavior change wheel) — a guide to designing interventions: Silverback Publishing; 2014. p. 329.

85. Lewis CC, Klasnja P, Powell BJ, Lyon AR, Tuzzio L, Jones S, et al. From classification to causality: advancing understanding of mechanisms of change in implementation science. Front Public Health [Internet]. 2018 [cited 2020 May 5];6. Available from: https://www.frontiersin.org/artic les/10.3389/fpubh.2018.00136/full

86. Lewis CC, Boyd MR, Walsh-Bailey C, Lyon AR, Beidas R, Mittman B, et al A systematic review of empirical studies examining mechanisms of implementation in health. Implement Sci. 2020;15(1):21.

87. Bosch M, van der Weijden T, Wensing M, Grol R. Tailoring quality improvement interventions to identified barriers: a multiple case analysis. J Eval Clin Pract. 2007;13(2):161-8.

88. Graham ID, Logan J, Harrison MB, Straus SE, Tetroe J, Caswell W, et al. Lost in knowledge translation: time for a map? J Contin Educ Heal Prof. 2006:26(1):13-24.

89. Simera I, Moher D, Hoey J, Schulz KF, Altman DG. The EQUATOR network and reporting guidelines: helping to achieve high standards in reporting health research studies. Maturitas. 2009;63(1):4-6.

90. CONSORT. Consort - Welcome to the CONSORT Website [Internet] [cited 2020 May 11]. Available from: http://www.consort-statement. org/

91. Hoffmann TC, Glasziou PP, Boutron I, Milne R, Perera R, Moher D, et al. Better reporting of interventions: template for intervention description and replication (TIDieR) checklist and guide. Res METHODS. 2014;12

92. Harrison MB, Graham ID, van den Hoek J, Dogherty EJ, Carley ME, Angus V. Guideline adaptation and implementation planning: a prospective observational study. Implement Sci IS. 2013;8:49.

93. Can-Implement@ planning for best-practice implementation. [Internet]. Philadelphia: Lippincott Williams \& Wilkins; 2014 [cited 2021 Sep 27] Available from: http://ezproxy.library.dal.ca/login?url=http://ovidsp. ovid.com/ovidweb.cgi?T=JS\&NEWS $=n \& C S C=Y \& P A G E=$ booktext $\& D=$ books\&AN=01833066\$\&PATH $=/ P G(0)$.

94. Leeman J, Birken SA, Powell BJ, Rohweder C, Shea CM. Beyond "implementation strategies": classifying the full range of strategies used in implementation science and practice. Implement Sci. 2017;12(1):125.
95. Damschroder LJ, Aron DC, Keith RE, Kirsh SR, Alexander JA, Lowery JC. Fostering implementation of health services research findings into practice: a consolidated framework for advancing implementation science. Implement Sci IS. 2009;4:50.

96. Dickerson J, Latina A. Team nursing: a collaborative approach improves patient care. Nursing 2021. 2017 Oct;47(10):16-7.

97. Lokker C, McKibbon KA, Colquhoun H, Hempel S. A scoping review of classification schemes of interventions to promote and integrate evidence into practice in healthcare. Implement Sci. 2015;10(1):27.

98. Slaughter SE, Zimmermann GL, Nuspl M, Hanson HM, Albrecht L, Esmail $\mathrm{R}$, et al. Classification schemes for knowledge translation interventions: a practical resource for researchers. BMC Med Res Methodol [Internet]. 2017 [cited 2021 mar 10];17(1):161. Available from: https://doi.org/10. 1186/s12874-017-0441-2

99. Albarracín D, Gillette JC, Earl AN, Glasman LR, Durantini MR, Ho M-H. A test of major assumptions about behavior change: a comprehensive look at the effects of passive and active HIV-prevention interventions since the beginning of the epidemic. Psychol Bull. 2005;131(6):856-97.

100. Tomasone JR, Kauffeldt KD, Chaudhary R, Brouwers MC. Effectiveness of guideline dissemination and implementation strategies on health care professionals' behaviour and patient outcomes in the cancer care context: a systematic review. Implement Sci. 2020;15(1):41.

101. Dadich A, Piper A, Coates D. Implementation science in maternity care: a scoping review. Implement Sci. 2021;16(1):16.

102. Meleis Al. Theoretical nursing: development and progress: Lippincott Williams \& Wilkins; 2011. p. 688.

\section{Publisher's Note}

Springer Nature remains neutral with regard to jurisdictional claims in published maps and institutional affiliations.

Ready to submit your research? Choose BMC and benefit from

- fast, convenient online submission

- thorough peer review by experienced researchers in your field

- rapid publication on acceptance

- support for research data, including large and complex data types

- gold Open Access which fosters wider collaboration and increased citations

- maximum visibility for your research: over 100M website views per year

At BMC, research is always in progress.

Learn more biomedcentral.com/submissions 